\title{
A minimization method and applications to the study of solitons
}

\author{
Vieri Benci* Donato Fortunato ${ }^{\dagger}$ \\ Dedicated to V. Lakshmikantham
}

November 3, 2018

\begin{abstract}
Roughly speaking a solitary wave is a solution of a field equation whose energy travels as a localized packet and which preserves this localization in time. A soliton is a solitary wave which exhibits some strong form of stability so that it has a particle-like behavior. In this paper, we prove a general, abstract theorem (Theorem 26) which allows to prove the existence of a class of solitons. Such solitons are suitable minimizers of a constrained functional and they are called hylomorphic solitons. Then we apply the abstract theory to problems related to the nonlinear Schrödinger equation (NSE) and to the nonlinear Klein-Gordon equation (NKG).
\end{abstract}

AMS subject classification: 47J30, 35J50, 35Q55, 35Q51, 37K45.

Key words: lack of compactness, orbital stability, nonlinear Schrödinger equation, lattice, nonlinear Klein-Gordon equation, solitary waves, hylomorphic solitons, vortices.

\section{Contents}

\section{Introduction}

2 A minimization result 2

2.1 The abstract framework . . . . . . . . . . . . . 3

2.2 The minimization theorem . . . . . . . . . . . . . . . 4

* Dipartimento di Matematica Applicata, Università degli Studi di Pisa, Via F. Buonarroti 1/c, Pisa, ITALY and Department of Mathematics, College of Science, King Saud University, Riyadh, 11451, SAUDI ARABIA. e-mail: benci@dma.unipi.it

${ }^{\dagger}$ Dipartimento di Matematica, Università degli Studi di Bari Aldo Moro, Via Orabona 4, 70125 Bari, Italy, e-mail: fortunat@dm.uniba.it 
3 An existence result of vortices for NSE 9

3.1 Statement of the problem . . . . . . . . . . . . . . . 10

3.2 Existence of vortices . . . . . . . . . . . . . . . . . . 12

4 Existence of hylomorphic solitons $\lcm{22}$

4.1 An abstract definition of solitary waves and solitons . . . . . . 22

4.2 An existence result for hylomorphic solitons . . . . . . . . . . 23

4.3 A stability result . . . . . . . . . . . . . . . 24

5 Existence of solitons for NSE with periodic potential 26

6 Existence of Solitons for the nonlinear Klein-Gordon equation 29

6.1 The NKG as a dynamical system . . . . . . . . . . . . . . 30

6.2 Existence results for NKG . . . . . . . . . . . . . . . . 31

\section{Introduction}

In some recent papers $(4,6],[9$, 14, 11], 12]) the existence of solitons has been proved using variational methods. In this paper, we prove a general, abstract theorem (Theorem 26) which applies to most of the situations analyzed in the mentioned papers.

The proof of Theorem 26 is carried out in two steps: in the first step (section 2) the research of the minimizers of a constrained functional is reduced to the study of the minimizers of a suitable free functional. The existence of such minimizers is stated in Theorem 8 . In the second step stability properties of these minimizers are proved (section 4).

These two theorems (Theorem 8 and Theorem 26) can be applied to the situations described in the quoted papers relative to solitons. In section 4 we give an abstract definition of soliton (Definition 22) and hylomorphic soliton (Definition 25). These solitons are stable minimizers of a constrained functional. Then we apply the abstract theorems 26 and 8 to new problems related to the nonlinear Schrödinger equation (NSE) and to the nonlinear Klein-Gordon equation (NKG): in section 3 we use Theorem 8 in order to prove the existence of vortices for the NSE with a potential $V$ which is periodic in one direction (Theorem [12). In section 5 we use Theorem 26 in order to prove the existence of hylomorphic solitons for NSE in a lattice, namely in presence of a periodic potential $V$.

Finally in section 6 we use Theorem 26 to prove the existence of hylomorphic solitons for the nonlinear Klein-Gordon equation (NKG).

\section{A minimization result}

Let $E$ and $C$ be two functionals on an Hilbert space $X$. We are interested in the following minimization problem: find values of $\sigma \in \mathbb{R}$, such that $E$ attains 
a constrained minimum on $\mathfrak{M}_{\sigma}$ where

$$
\mathfrak{M}_{\sigma}:=\{\mathbf{u} \in X|| C(\mathbf{u}) \mid=\sigma\} .
$$

In the next section, we will describe the abstract framework where we will work and then we will prove an abstract existence theorem.

\subsection{The abstract framework}

We need some definitions. These definitions are related to a couple $(X, G)$ where $G$ is a group acting on the Hilbert space $X$. In our applications $G$ will be a subgroup of the group of translations.

Definition 1 A non-empty subset $\Gamma \subset X$ is called $G$-invariant if

$$
\forall \mathbf{u} \in \Gamma, \forall g \in G, g \mathbf{u} \in \Gamma .
$$

Definition 2 A functional $J$ on $X$ is called $G$-invariant if

$$
\forall g \in G, \forall \mathbf{u} \in X, J(g \mathbf{u})=J(\mathbf{u})
$$

Definition 3 A closed $G$-invariant set $\Gamma \subset X$ is called $G$-compact if for any sequence $\mathbf{u}_{n}$ in $\Gamma$ there is a sequence $g_{n} \in G$, such that $g_{n} \mathbf{u}_{n}$ has a converging subsequence. Clearly a sequence $\mathbf{u}_{n}$ in $X$ will be called $G$-compact if its image is G-compact.

Definition $4 A G$-invariant functional $J$ on $X$ is called $G$-compact if any minimizing sequence $\mathbf{u}_{n}$ is $G$-compact.

Clearly a $G$-compact functional has a $G$-compact set of minimizers.

In order to prove an existence result for the minimizers of $E$ on $\mathfrak{M}_{\sigma}$, we impose some assumptions to $E$ and $C$; to do this we need some other definitions:

Definition 5 (Splitting property)We say that a functional $F$ on $X$ has the splitting property if given a sequence $\mathbf{u}_{n}=\mathbf{u}+\mathbf{w}_{n}$ in $X$ such that $\mathbf{w}_{n}$ converges weakly to 0 , we have that

$$
F\left(\mathbf{u}_{n}\right)=F(\mathbf{u})+F\left(\mathbf{w}_{n}\right)+o(1) .
$$

Remark 6 A symmetric, continuous quadratic form satisfies the splitting property; in fact, in this case, we have that $F(\mathbf{u}):=\langle L \mathbf{u}, \mathbf{u}\rangle$ for some continuous selfajoint operator $L$; then given a sequence $\mathbf{u}_{n}=\mathbf{u}+\mathbf{w}_{n}$ with $\mathbf{w}_{n} \rightarrow 0$ weakly, we have that

$$
\begin{aligned}
F\left(\mathbf{u}_{n}\right) & =\langle L \mathbf{u}, \mathbf{u}\rangle+\left\langle L \mathbf{w}_{n}, \mathbf{w}_{n}\right\rangle+2\left\langle L \mathbf{u}, \mathbf{w}_{n}\right\rangle \\
& =F(\mathbf{u})+F\left(\mathbf{w}_{n}\right)+o(1) .
\end{aligned}
$$

Now we can formulate the required properties on $E$ and $C$ : 
- (EC-1) (Value at 0) $E, C$ are $C^{1}$, bounded functionals such that

$$
E(0)=0, C(0)=0 ; E^{\prime}(0)=0 ; C^{\prime}(0)=0 .
$$

- (EC-2) (Invariance) $E$ and $C$ are $G$-invariant.

- (EC-3)(Coercivity) We distinguish two cases: $C \geq 0$ and $C$ not positive. If $C \geq 0$ we assume that there exists $a \geq 0$ and $s \geq 1$ such that

- (i) $E(\mathbf{u})+a C(\mathbf{u})^{s} \geq 0$;

- (ii) if $\|\mathbf{u}\| \rightarrow \infty$, then $E(\mathbf{u})+a C(\mathbf{u})^{s} \rightarrow \infty$;

- (iii) for any bounded sequence $\mathbf{u}_{n}$ in $X$ such that $E\left(\mathbf{u}_{n}\right)+a C\left(\mathbf{u}_{n}\right)^{s} \rightarrow$ 0 , we have that $\mathbf{u}_{n} \rightarrow 0$.

In the case in which $C$ is not positive we assume that (i), (ii), (iii) hold true with $a=0$.

- $(E C-4)($ Splitting property) $E$ and $C$ satisfy the splitting property.

Before stating our main theorem we need this definition:

Definition $7 \quad A$ bounded sequence $\mathbf{u}_{n}$ in $X$ is called vanishing sequence if for any subsequence $\mathbf{u}_{n_{k}}$ and any sequence $g_{k} \subset G, g_{k} \mathbf{u}_{n_{k}}$ converges weakly to 0 .

Observe that the notion of vanishing sequence depends on the group $G$ acting on $X$. Clearly a bounded sequence $\mathbf{u}_{n}$ in $X$ is a non-vanishing sequence if there exists a subsequence $\mathbf{u}_{n_{k}}$ and a sequence $g_{k} \subset G$, such that $g_{k} \mathbf{u}_{n_{k}}$ converges weakly to some $\mathbf{u} \neq 0$.

So, if $\mathbf{u}_{n} \rightarrow 0$ strongly, then $\mathbf{u}_{n}$ is a vanishing sequence. However, if $\mathbf{u}_{n} \rightarrow 0$ weakly, it might happen that it is a non-vanishing sequence.

\subsection{The minimization theorem}

We now set

$$
\Lambda(\mathbf{u}):=\frac{E(\mathbf{u})}{|C(\mathbf{u})|}
$$

and

$$
\Lambda_{0}:=\inf \left\{\liminf \Lambda\left(\mathbf{u}_{n}\right) \mid \mathbf{u}_{n} \text { is a vanishing sequence }\right\} .
$$

Now we state and prove the following existence result:

Theorem 8 Let $E$ and $C$ be two functionals on a Hilbert space $X$ and $G$ be a group acting on $X$. Assume that $E$ and $C$ satisfy $(E C-1), \ldots,(E C-4)$ and

$$
\inf \Lambda(\mathbf{u})<\Lambda_{0} .
$$


Then there is $\bar{\delta}>0$ and a family of values $c_{\delta}, \delta \in(0, \bar{\delta})$, such that the minimum

$$
e_{\delta}=\min \left\{E(\mathbf{u})|| C(\mathbf{u}) \mid=c_{\delta}\right\}
$$

exists and the set $\Gamma_{c_{\delta}}$ of minimizers is $G$-compact. Moreover $\Gamma_{c_{\delta}}$ can be characterized as the set of minimizers of the functional

$$
J_{\delta}(\mathbf{u})=\Lambda(\mathbf{u})+\delta \Phi(\mathbf{u}) \delta \in(0, \bar{\delta})
$$

where $\Phi(\mathbf{u})=E(\mathbf{u})+2 a|C(\mathbf{u})|^{s}$ with a as in (EC-3).

Remark 9 When we will apply Th. 8, it is necessary to estimate $\Lambda_{0}$; the following inequalities may help to do this. In order to give an estimate from below, assume that there exists a seminorm $\|\mathbf{u}\|_{\sharp}$ such that

$$
\left\{\mathbf{u}_{n} \text { is a vanishing sequence }\right\} \Rightarrow\left\|\mathbf{u}_{n}\right\|_{\sharp} \rightarrow 0 \text {. }
$$

Then we have that

$$
\Lambda_{0} \geq \liminf _{\|\mathbf{u}\|_{\sharp} \rightarrow 0} \Lambda(\mathbf{u}) .
$$

Let \|\| denote the norm in $X$. Since

$$
\left\|\mathbf{u}_{n}\right\| \rightarrow 0 \Rightarrow\left\{\mathbf{u}_{n} \text { is a vanishing sequence }\right\},
$$

we have that

$$
\Lambda_{0} \leq \liminf _{\|\mathbf{u}\| \rightarrow 0} \Lambda(\mathbf{u}) .
$$

Moreover, if $E$ and $C$ are twice differentiable in 0, by (EC-O), we have that

$$
\begin{aligned}
\liminf _{\|\mathbf{u}\| \rightarrow 0} \Lambda(\mathbf{u}) & =\liminf _{\|\mathbf{u}\| \rightarrow 0} \frac{E(0)+E^{\prime}(0)[\mathbf{u}]+E^{\prime \prime}(0)[\mathbf{u}, \mathbf{u}]+o\left(\|\mathbf{u}\|^{2}\right)}{\left|C(0)+C^{\prime}(0)[\mathbf{u}]+C^{\prime \prime}(0)[\mathbf{u}, \mathbf{u}]+o\left(\|\mathbf{u}\|^{2}\right)\right|} \\
& =\inf \frac{E^{\prime \prime}(0)[\mathbf{u}, \mathbf{u}]}{\left|C^{\prime \prime}(0)[\mathbf{u}, \mathbf{u}]\right|}
\end{aligned}
$$

and hence

$$
\Lambda_{0} \leq \inf \frac{E^{\prime \prime}(0)[\mathbf{u}, \mathbf{u}]}{\left|C^{\prime \prime}(0)[\mathbf{u}, \mathbf{u}]\right|}
$$

It is can be seen that in many applications the two limits (6) and (7) coincide and in this case we get a sharp estimate for $\Lambda_{0}$.

Remark 10 If $X=H^{1}\left(\mathbb{R}^{N}\right)$ and $G=\mathbb{Z}^{N}$ with the action 94), then (see Proposition [32) the norm $\|\mathbf{u}\|_{\sharp}=\|\mathbf{u}\|_{L^{t}}, t \in\left(2, \frac{2 N}{N-2}\right), N \geq 3$, satisfies the property (5) of the preceding remark 9 and consequently it satisfies also (6)).

Remark 11 The fact that the minimization problem of $E$ on $\mathfrak{M}_{\sigma}$ reduces to the minimization of a free functional $J_{\delta}(\mathbf{u})$ is very useful in numerical simulation. 
Proof of theorem 8, By (3) there exists $\mathbf{v} \in X$ such that $\Lambda(\mathbf{v})<\Lambda_{0}$, then we can take $\delta>0$ so small that

$$
J_{\delta}(\mathbf{v})=\Lambda(\mathbf{v})+\delta \Phi(\mathbf{v})<\Lambda_{0}
$$

and define

$$
\bar{\delta}=\sup \left\{\delta \mid \exists \mathbf{v}: \Lambda(\mathbf{v})+\delta \Phi(\mathbf{v})<\Lambda_{0}\right\} .
$$

Then

$$
\inf _{\mathbf{u} \in X} J_{\delta}(\mathbf{u})<\Lambda_{0} \text { for } \delta \in(0, \bar{\delta})
$$

Now we show that

$$
J_{\delta}(\mathbf{u}) \geq \frac{\delta}{2} \Phi(\mathbf{u})-M
$$

where $M$ is a suitable constant. Clearly, if $C$ is not positive, we have $a=0$ in (EC-3)(i); then $\Phi(\mathbf{u})=E(\mathbf{u}) \geq 0$ and $J_{\delta}(\mathbf{u})=\frac{E(\mathbf{u})}{|C(\mathbf{u})|}+\delta E(\mathbf{u})$. Then (9) is obviously satisfied.

Now assume that $C(\mathbf{u}) \geq 0$. By (EC-3)(i) we have that

$$
E(\mathbf{u}) \geq-a C(\mathbf{u})^{s}
$$

and hence

$$
\frac{E(\mathbf{u})}{C(\mathbf{u})} \geq-a C(\mathbf{u})^{s-1}
$$

Then, by (10) and (11), we get

$$
\begin{aligned}
J_{\delta}(\mathbf{u}) & =\frac{E(\mathbf{u})}{C(\mathbf{u})}+\delta \Phi(\mathbf{u}) \geq-a C(\mathbf{u})^{s-1}+\frac{\delta}{2}\left[E(\mathbf{u})+2 a C(\mathbf{u})^{s}\right]+\frac{\delta}{2} \Phi(\mathbf{u}) \\
& \geq-a C(\mathbf{u})^{s-1}+\frac{\delta}{2}\left[-a C(\mathbf{u})^{s}+2 a C(\mathbf{u})^{s}\right]+\frac{\delta}{2} \Phi(\mathbf{u}) \\
& \geq-a C(\mathbf{u})^{s-1}+\frac{a \delta}{2} C(\mathbf{u})^{s}+\frac{\delta}{2} \Phi(\mathbf{u}) \geq \frac{\delta}{2} \Phi(\mathbf{u})-M
\end{aligned}
$$

where

$$
M=-a \min _{t \geq 0}\left(\frac{\delta}{2} t^{s}-t^{s-1}\right) .
$$

Then (9) has been proved.

Now let us prove that $J_{\delta}$ is $G$ - compact (see Definition 4).

Let $\mathbf{u}_{n}$ be a minimizing sequence of $J_{\delta}$. This sequence $\mathbf{u}_{n}$ is bounded in $X$. In fact, arguing by contradiction, assume that, up to a subsequence, $\left\|\mathbf{u}_{n}\right\| \rightarrow \infty$. Then by (9) and (EC-3) (ii) we get $J_{\delta}\left(\mathbf{u}_{n}\right) \rightarrow \infty$ which contradicts the fact that $\mathbf{u}_{n}$ is a minimizing sequence of $J_{\delta}$.

Since $\mathbf{u}_{n}$ is minimizing for $J_{\delta}$, by (죠 , there exists $\eta>0$ such that, for $n$ sufficiently large,

$$
\frac{E\left(\mathbf{u}_{n}\right)}{\left|C\left(\mathbf{u}_{n}\right)\right|}+\delta \Phi\left(\mathbf{u}_{n}\right)<\Lambda_{0}-\eta
$$


then

$$
\Lambda\left(\mathbf{u}_{n}\right)=\frac{E\left(\mathbf{u}_{n}\right)}{\left|C\left(\mathbf{u}_{n}\right)\right|}<\Lambda_{0}-\eta
$$

On the other hand

$$
\Lambda\left(\mathbf{u}_{n}\right) \text { is bounded below. }
$$

In fact: since $\Phi$ is bounded and $\mathbf{u}_{n}$ is bounded in $X, \Phi\left(\mathbf{u}_{n}\right)$ is bounded. So we deduce from (9) that $J_{\delta}\left(\mathbf{u}_{n}\right)$ is bounded below. Then $\Lambda\left(\mathbf{u}_{n}\right)=J_{\delta}\left(\mathbf{u}_{n}\right)-\delta \Phi\left(\mathbf{u}_{n}\right)$ is bounded below and so (13) is proved. By (12) and (13) we have, for some subsequence, that

$$
\Lambda\left(\mathbf{u}_{n}\right) \rightarrow \lambda, \quad-\infty<\lambda<\Lambda_{0} .
$$

Then, by (21), $\mathbf{u}_{n}$ is a bounded non vanishing sequence. Hence, by Def. 7 we can extract a subsequence $\mathbf{u}_{n_{k}}$ and we can take a sequence $g_{k} \subset G$ such that $\mathbf{u}_{k}^{\prime}:=g_{k} \mathbf{u}_{n_{k}}$ is weakly convergent to some

$$
\overline{\mathbf{u}} \neq 0 .
$$

We can write

$$
\mathbf{u}_{n}^{\prime}=\overline{\mathbf{u}}+\mathbf{w}_{n}
$$

with $\mathbf{w}_{n} \rightarrow 0$ weakly. We want to prove that $\mathbf{w}_{n} \rightarrow 0$ strongly. First of all we will show that

$$
\lim \Phi\left(\overline{\mathbf{u}}+\mathbf{w}_{n}\right) \geq \Phi(\overline{\mathbf{u}})+\lim \Phi\left(\mathbf{w}_{n}\right) .
$$

If $C(\mathbf{u})$ is not positive we have $a=0$ in (EC-3)(i), then $\Phi(\mathbf{u})=E(\mathbf{u})$ and clearly (16) holds as an equality since $E$ satisfies assumption (EC-4) (splitting property).

Now assume that $C(\mathbf{u}) \geq 0$. Then by (EC-4) and since $s \geq 1$, we have that

$$
\begin{aligned}
\lim \Phi\left(\overline{\mathbf{u}}+\mathbf{w}_{n}\right) & =\lim \left(E\left(\overline{\mathbf{u}}+\mathbf{w}_{n}\right)+2 a C\left(\overline{\mathbf{u}}+\mathbf{w}_{n}\right)^{s}\right) \\
& =E(\overline{\mathbf{u}})+\lim E\left(\mathbf{w}_{n}\right)+2 a \lim \left(C(\overline{\mathbf{u}})+C\left(\mathbf{w}_{n}\right)\right)^{s} \\
& \geq E(\overline{\mathbf{u}})+\lim E\left(\mathbf{w}_{n}\right)+2 a \lim \left(C(\overline{\mathbf{u}})^{s}+C\left(\mathbf{w}_{n}\right)^{s}\right) \\
& =E(\overline{\mathbf{u}})+2 a C(\overline{\mathbf{u}})^{s}+\lim E\left(\mathbf{w}_{n}\right)+2 a \lim C\left(\mathbf{w}_{n}\right)^{s} \\
& =\Phi(\overline{\mathbf{u}})+\lim \Phi\left(\mathbf{w}_{n}\right) .
\end{aligned}
$$

So (16) has been proved.

Next we show that

$$
C\left(\overline{\mathbf{u}}+\mathbf{w}_{\mathbf{n}}\right) \text { does not converge to } 0 .
$$

Arguing by contradiction assume that $C\left(\overline{\mathbf{u}}+\mathbf{w}_{\mathbf{n}}\right)$ converges to 0 . Then, since $\overline{\mathbf{u}}+\mathbf{w}_{\mathbf{n}}$ is a minimizing sequence for $J_{\delta}$, also $E\left(\overline{\mathbf{u}}+\mathbf{w}_{\mathbf{n}}\right)$ converges to 0 and then

$$
E\left(\overline{\mathbf{u}}+\mathbf{w}_{\mathbf{n}}\right)+a\left|C\left(\overline{\mathbf{u}}+\mathbf{w}_{\mathbf{n}}\right)\right|^{s} \rightarrow 0 .
$$

So, by (EC-3)(iii), we get

$$
\overline{\mathbf{u}}+\mathbf{w}_{\mathbf{n}} \rightarrow 0 \text { in } X .
$$


From (19) and since $\mathbf{w}_{\mathbf{n}} \rightarrow 0$ weakly in $X$, we have that $\overline{\mathbf{u}}=0$, contradicting (15). Then (18) holds and consequently, up to a subsequence, we have

$$
\left|C\left(\overline{\mathbf{u}}+\mathbf{w}_{\mathbf{n}}\right)\right|=\left|C(\overline{\mathbf{u}})+C\left(\mathbf{w}_{n}\right)+o(1)\right| \geq \text { const. }>0 .
$$

Now, we set

$$
\begin{aligned}
& j_{\delta}=\inf J_{\delta}=\lim J_{\delta}\left(\mathbf{u}_{n}^{\prime}\right) ; e_{\delta}=E(\overline{\mathbf{u}}) ; c_{\delta}=|C(\overline{\mathbf{u}})| \\
& e_{1}=\lim E\left(\mathbf{w}_{n}\right) ; c_{1}=\lim \left|C\left(\mathbf{w}_{n}\right)\right| .
\end{aligned}
$$

Observe that the $\operatorname{limits} \lim E\left(\mathbf{w}_{n}\right)$ and $\lim \left|C\left(\mathbf{w}_{n}\right)\right|$ exist (up to subsequences), since $E$ and $C$ are bounded functionals and $\mathbf{w}_{n}$ weakly converges.

Now we have

$$
\lim \frac{E(\overline{\mathbf{u}})+E\left(\mathbf{w}_{n}\right)+o(1)}{\left|C(\overline{\mathbf{u}})+C\left(\mathbf{w}_{n}\right)+o(1)\right|} \geq \frac{e_{\delta}+e_{1}}{c_{\delta}+c_{1}} .
$$

In fact, as usual we distinguish two cases: if $C \geq 0$ (21) holds since

$$
\lim \left(C(\overline{\mathbf{u}})+C\left(\mathbf{w}_{n}\right)+o(1)\right)=c_{\delta}+c_{1} .
$$

On the other hand, if $C$ is not positive, by (EC-3)(i), we have $E \geq 0$ and in this case

$$
\lim \left|C(\overline{\mathbf{u}})+C\left(\mathbf{w}_{n}\right)+o(1)\right| \leq c_{\delta}+c_{1} .
$$

So (21) holds also when $C$ is not positive.

Now by (21) and (16), we have that

$$
\begin{aligned}
j_{\delta} & =\lim \left[\frac{E\left(\mathbf{u}_{n}^{\prime}\right)}{\left|C\left(\mathbf{u}_{n}^{\prime}\right)\right|}+\delta \Phi\left(\mathbf{u}_{n}^{\prime}\right)\right] \\
& =\lim \frac{E(\overline{\mathbf{u}})+E\left(\mathbf{w}_{n}\right)+o(1)}{\left|C(\overline{\mathbf{u}})+C\left(\mathbf{w}_{n}\right)+o(1)\right|}+\delta \lim \Phi\left(\overline{\mathbf{u}}+\mathbf{w}_{n}\right) \\
& \geq \frac{e_{\delta}+e_{1}}{c_{\delta}+c_{1}}+\delta \lim \Phi\left(\mathbf{w}_{n}\right)+\delta \Phi(\overline{\mathbf{u}}) .
\end{aligned}
$$

Now we want to prove that

$$
\frac{e_{1}}{c_{1}} \geq \frac{e_{\delta}}{c_{\delta}} .
$$

We argue indirectly and we suppose that

$$
\frac{e_{\delta}}{c_{\delta}}>\frac{e_{1}}{c_{1}} .
$$

By the above inequality it follows that

$$
\frac{e_{\delta}+e_{1}}{c_{\delta}+c_{1}}=\frac{\frac{e_{\delta}}{c_{\delta}} c_{\delta}+\frac{e_{1}}{c_{1}} c_{1}}{c_{\delta}+c_{1}}>\frac{\frac{e_{1}}{c_{1}} c_{\delta}+\frac{e_{1}}{c_{1}} c_{1}}{c_{\delta}+c_{1}}=\frac{e_{1}}{c_{1}}
$$

and hence, by (22) and (25), we get

$$
j_{\delta} \geq \frac{e_{\delta}+e_{1}}{c_{\delta}+c_{1}}+\delta \lim \Phi\left(\mathbf{w}_{n}\right)+\delta \Phi(\overline{\mathbf{u}})
$$




$$
\begin{gathered}
>\frac{e_{1}}{c_{1}}+\delta \lim \Phi\left(\mathbf{w}_{n}\right)+\delta \Phi(\overline{\mathbf{u}})=\lim J_{\delta}\left(\mathbf{w}_{n}\right)+\delta \Phi(\overline{\mathbf{u}}) \\
\geq \inf J_{\delta}+\delta \Phi(\overline{\mathbf{u}})=j_{\delta}+\delta \Phi(\overline{\mathbf{u}}) \geq j_{\delta} .
\end{gathered}
$$

So we get a contradiction and (24) cannot occur. Then we have (23).

Now, by (23), we get

$$
\frac{e_{\delta}+e_{1}}{c_{\delta}+c_{1}}=\frac{\frac{e_{\delta}}{c_{\delta}} c_{\delta}+\frac{e_{1}}{c_{1}} c_{1}}{c_{\delta}+c_{1}} \geq \frac{\frac{e_{\delta}}{c_{\delta}} c_{\delta}+\frac{e_{\delta}}{c_{\delta}} c_{1}}{c_{\delta}+c_{1}}=\frac{e_{\delta}}{c_{\delta}} .
$$

So we get

$$
\frac{e_{\delta}+e_{1}}{c_{\delta}+c_{1}} \geq \frac{e_{\delta}}{c_{\delta}}
$$

Then, using (22), the above inequality and the fact that $j_{\delta}=\inf J_{\delta}$, we get

$$
\begin{aligned}
j_{\delta} & \geq \frac{e_{\delta}+e_{1}}{c_{\delta}+c_{1}}+\delta \Phi(\overline{\mathbf{u}})+\delta \lim \Phi\left(\mathbf{w}_{n}\right) \\
& \geq \frac{e_{\delta}}{c_{\delta}}+\delta \Phi(\overline{\mathbf{u}})+\delta \lim \Phi\left(\mathbf{w}_{n}\right) \\
& =J_{\delta}(\overline{\mathbf{u}})+\delta \lim \Phi\left(\mathbf{w}_{n}\right) \geq j_{\delta}+\delta \lim \Phi\left(\mathbf{w}_{n}\right) .
\end{aligned}
$$

Then

$$
\delta \lim \Phi\left(\mathbf{w}_{n}\right) \leq 0
$$

and, by (EC-3)(iii), $\mathbf{w}_{n} \rightarrow 0$ and hence $\mathbf{u}_{n}^{\prime} \rightarrow \overline{\mathbf{u}}$ strongly and $\overline{\mathbf{u}}$ is a minimizer of $J_{\delta}$.

So we conclude that $J_{\delta}$ is $G$-compact.

Since $\overline{\mathbf{u}}$ is a minimizer of $J_{\delta}$, clearly $\overline{\mathbf{u}}$ minimizes also the functional

$$
\frac{E(\mathbf{u})}{c_{\delta}}+\delta\left[E(\mathbf{u})+a c_{\delta}^{s}\right]=\left(\frac{1}{c_{\delta}}+\delta\right) E(\mathbf{u})+\delta a c_{\delta}^{s}
$$

on the set $\left\{\mathbf{u} \in X|| C(\mathbf{u}) \mid=c_{\delta}\right\}$ and hence $\overline{\mathbf{u}}$ minimizes also $E$ on this set. Now denote by $\Gamma_{c_{\delta}}$ the set of such minimizers. It is easy to see that viceversa $\Gamma_{c_{\delta}}$ is contained in the set of minimizers of $J_{\delta}$. So, since $J_{\delta}$ is $G$-compact, we conclude that $\Gamma_{c_{\delta}}$ is $G$-compact.

\section{An existence result of vortices for NSE}

The existence of vortices is an interesting and old issue in many questions of mathematical physics as superconductivity, classical and quantum field theory, string and elementary particle theory (see the pioneering papers [1, 25] and e.g. the more recent ones [24, 29], 30], 28] with their references).

From mathematical viewpoint, the existence of vortices for the nonlinear Klein-Gordon equations (NKG), for nonlinear Schroedinger equations (NSE) 
and for nonlinear Klein-Gordon-Maxwell equations (NKGM) has been studied in some recent papers ( [18, 2], 3], 10, 16, [7, 8, 15).

Many of the previous results can be obtained applying Th8 Here we will consider a case not covered by the existing literature, namely the study of vortices in NSE when the potential $V(x)$ depends only on the third variable and it is periodic, namely for all $k \in \mathbb{Z}$

$$
V\left(x_{1}, x_{2}, x_{3}\right)=V\left(x_{3}\right)=V\left(x_{3}+k\right) .
$$

\subsection{Statement of the problem}

Let us consider the nonlinear Schroedinger equation:

$$
i \frac{\partial \psi}{\partial t}=-\frac{1}{2} \Delta \psi+\frac{1}{2} W^{\prime}(\psi)+V(x) \psi .
$$

where $\psi(t, x)$ is a complex valued function defined on the space-time $\mathbb{R} \times \mathbb{R}^{N}$ $(N \geq 3), V: \mathbb{R}^{N} \rightarrow \mathbb{R}, W: \mathbb{C} \rightarrow \mathbb{R}$ such that $W(\psi)=F(|\psi|)$ for some smooth function $F:[0, \infty) \rightarrow \mathbb{R}$ and

$$
W^{\prime}(\psi)=\frac{\partial W}{\partial \psi_{1}}+i \frac{\partial W}{\partial \psi_{2}}, \quad \psi=\psi_{1}+i \psi_{2}
$$

namely

$$
W^{\prime}(\psi)=F^{\prime}(|\psi|) \frac{\psi}{|\psi|} .
$$

Equation (NSE) is the Euler-Lagrange equation relative to the Lagrangian density

$$
\mathcal{L}=\operatorname{Re}\left(i \partial_{t} \psi \bar{\psi}\right)-\frac{1}{2}|\nabla \psi|^{2}-V(x)|\psi|^{2}-W(\psi) .
$$

By the well known Noether's theorem (see e.g. [22, 9]) the invariance of $\mathcal{L}$ under a one parameter Lie group gives rise to a constant of the motion.

Since $\mathcal{L}$ is invariant under the action of the time translations the energy

$$
\mathcal{E}(\psi)=\frac{1}{2} \int\left[|\nabla \psi|^{2}+V(x)|\psi|^{2}\right] d x+\int W(\psi)
$$

is constant along the solutions of (NSE).

Since $W(\psi)=F(|\psi|), \mathcal{L}$ is invariant under the $S^{1}$ action

$$
\psi \rightarrow e^{i \theta} \psi
$$

then the charge $C(\psi)$, defined by

$$
C(\psi)=\int|\psi|^{2},
$$

is constant along the solutions of (NSE) (see e.g. [9]). 
The angular momentum, by definition, is the quantity which is preserved by virtue of the invariance under space rotations (with respect to the origin) of the Lagrangian. We shall consider, for simplicity, the case of three space dimensions $N=3$. If we assume that

$$
V\left(x_{1}, x_{2}, x_{3}\right)=V\left(x_{3}\right)
$$

namely that $V$ depends only on the third coordinate, then the Lagrangian is invariant under the group of rotations around the axis $x_{3}$. In this case the third component of the momentum

$$
M_{3}(\psi)=\operatorname{Re} \int\left(x_{1} \partial_{x_{2}} \psi-x_{2} \partial_{x_{1}} \psi\right) d x
$$

is a constant of motion. Using the polar form

$$
\psi(t, x)=u(t, x) e^{i S(t, x)}, u \geq 0
$$

$M_{3}(\psi)$ can be written as follows

$$
M_{3}(\psi)=\int\left(x_{1} \partial_{x_{2}} S-x_{2} \partial_{x_{1}} S\right) u^{2} d x .
$$

A solution of (NSE) is called standing wave if it has the following form:

$$
\psi(t, x)=\psi_{0}(x) e^{-i \omega t}, \quad \omega>0
$$

A vortex is a standing wave with nonvanishing angular momentum.

It is immediate to check that if $\psi_{0}(x)$ in (30) has real values, the angular momentum $M_{3}(\psi)$ is trivial. However, if $\psi_{0}(x)$ is allowed to have complex values, it is possible to have $M_{3}(\psi) \neq 0$. Thus, we are led to make an ansatz of the following form:

$$
\psi(t, x)=u(x) e^{i(\ell \theta(x)-\omega t)}, \quad u(x) \geq 0, \omega \in \mathbb{R}, \ell \in \mathbb{Z}-\{0\}
$$

and

$$
\theta(x)=\operatorname{Im} \log \left(x_{1}+i x_{2}\right) \in \mathbb{R} / 2 \pi \mathbb{Z} ; \quad x=\left(x_{1}, x_{2}, x_{3}\right) .
$$

Moreover, we assume that $u$ has a cylindrical symmetry, namely

$$
u(x)=u\left(r, x_{3}\right), \text { where } r=\sqrt{x_{1}^{2}+x_{2}^{2}} .
$$

By this ansatz, equation (NSE) is equivalent to the system

$$
\left\{\begin{array}{l}
-\triangle u+\ell^{2}|\nabla \theta|^{2} u+W^{\prime}(u)+2 V\left(x_{3}\right) \psi=2 \omega u \\
u^{2} \triangle \theta+2 \nabla u \cdot \nabla \theta=0 .
\end{array}\right.
$$

By the definition of $\theta$ and (32) we have

$$
\triangle \theta=0, \quad \nabla \theta \cdot \nabla u=0, \quad|\nabla \theta|^{2}=\frac{1}{r^{2}}
$$


where the dot · denotes the euclidean scalar product.

So the above system reduces to find solutions, with symmetry (32), of the equation

$$
-\triangle u+\frac{\ell^{2}}{r^{2}} u+W^{\prime}(u)+2 V(x) u=2 \omega u \quad \text { in } \mathbb{R}^{3} .
$$

Direct computations show that the energy and the third component of the angular momentum become

$$
\begin{aligned}
E_{\ell}(u)= & \mathcal{E}\left(u(x) e^{i(\ell \theta(x)-\omega t)}\right) \\
= & \int_{\mathbb{R}^{3}}\left[\frac{1}{2}|\nabla u|^{2}+\left(\frac{1}{2} \frac{\ell^{2}}{r^{2}}+V(x)\right) u^{2}+W(u)\right] d x \\
& M_{3}\left(u(x) e^{i(\ell \theta(x)-\omega t)}\right)=-\ell \int_{\mathbb{R}^{3}} u^{2} d x .
\end{aligned}
$$

We point out that $M_{3}$ in (36) is nontrivial when both $\ell$ and $u$ are not zero. Let us remark that the solutions of equation (33) can be obtained as critical points of the functional (34) on the manifold

$$
\mathfrak{M}_{c}:=\{u \in X \mid C(u)=c\}
$$

where $X$ is the Hilbert space obtained by the closure of $\mathcal{D}\left(\mathbb{R}^{N}\right) \sqrt{1}$ with respect to the norm

$$
\|u\|_{X}^{2}=\int_{\mathbb{R}^{3}}\left[|\nabla u|^{2}+\left(\frac{\ell}{r^{2}}+1\right) u^{2}\right] d x .
$$

Thus we can apply the minimization result (Theorem 8) stated in section 2 Cleary, using this approach, $2 \omega$ will be the Lagrange multiplier.

\subsection{Existence of vortices}

Recall that $W(\psi)=F(|\psi|)$. With abuse of notation, in the following we write $W$ instead of $F$ We make the following assumptions:

(i) $W: \mathbb{R}_{+} \rightarrow \mathbb{R}$ is a $C^{2}$ function which satisfies the following assumptions:

$$
\begin{gathered}
W(0)=W^{\prime}(0)=W^{\prime \prime}(0)=0 \\
\left|W^{\prime}(s)\right| \leq c_{1} s^{r-1}+c_{2} s^{q-1} \text { for } q, r \text { in }\left(2,2^{*}\right), 2^{*}=\frac{2 N}{N-2}, N \geq 3 \\
W(s) \geq-c s^{p}, c \geq 0,2<p<2+\frac{4}{N} \text { for } s \text { large } \\
\exists s_{0} \in \mathbb{R}^{+} \text {such that } \frac{W\left(s_{0}\right)}{s_{0}^{2}}<\inf V-\sup V
\end{gathered}
$$

\footnotetext{
${ }^{1} \mathcal{D}\left(\mathbb{R}^{N}\right)$ denotes the space of the infinitely differentiable functions with compact support defined in $\mathbb{R}^{N}$.
} 
(ii) $V: \mathbb{R}^{N} \rightarrow \mathbb{R}$ is a continuous function which satisfies the following assumptions:

$$
\begin{gathered}
1 \leq V(x) \leq V_{0}<\infty ; \\
\forall k \in \mathbb{Z}, V(x)=V\left(x_{3}\right)=V\left(x_{3}+k\right) .
\end{gathered}
$$

We get the following theorem:

Theorem 12 Assume that $\left(W_{0}\right), \ldots,\left(W_{3}\right)$ and $\left(V_{0}\right),\left(V_{1}\right)$, are satisfied. Then, for any integer $\ell \neq 0$, there exist $\bar{\delta}>0$ and a family $\psi_{\delta}, \delta \in(0, \bar{\delta})$, of vortices of (NSE) with angular momentum $\left(0,0,-\ell \int_{\mathbb{R}^{3}}\left|\psi_{\delta}\right|^{2} d x\right)$.

Remark 13 The conditions $\left(W_{0}\right)$ and $\left(V_{0}\right)$ are assumed for simplicity; in fact they can be easily weakened as follows

$$
W(0)=W^{\prime}(0)=0, \quad W^{\prime \prime}(0)=E_{0}
$$

and

$$
E_{1} \leq V(x) \leq V_{0}<+\infty .
$$

In fact, in the general case, it is possible to replace $W(s)$ with

$$
W_{1}(s)=W(s)-\frac{1}{2} E_{0} s^{2}
$$

and $V(x)$ with

$$
V_{1}(x)=V(x)-E_{1}+1 .
$$

In this case equation (33) becomes

$$
-\triangle u+\frac{\ell^{2}}{r^{2}} u+W_{1}^{\prime}(u)+2 V_{1}(x) u=\left(-E_{0}-2 E_{1}+2+2 \omega\right) u \quad \text { in } \mathbb{R}^{3} .
$$

Thus in the general case, there is only a change of the lagrange multiplier and so the solution of the Schroedinger equation is modified only by a phase factor.

By the preceding subsection we deduce that the existence of vortices of angular momentum $\ell$ is reduced to find critical points, having the symmetry (32), of the functional $E_{\ell}$ (34) on $\mathfrak{M}_{c}$.

Now consider the action $T_{\theta}$ of the group $S^{1}$ on $u\left(x_{1}, x_{2}, x_{3}\right) \in X$, defined by

$$
T_{\theta} u=u\left(R_{\theta}\left(x_{1}, x_{2}\right), x_{3}\right), \quad \theta \in \frac{\mathbb{R}}{2 \pi \mathbb{Z}},
$$

where $R_{\theta}$ denotes the rotation of an angle $\theta$ in the plane $x_{1}, x_{2}$. We set

$$
X_{r}=\left\{u \in X \mid u=u\left(r, x_{3}\right)\right\}, \mathfrak{M}_{c}^{r}=\mathfrak{M}_{c} \cap X_{r} .
$$

Observe that $V$ depends only on $x_{3}$, then the functional $E_{\ell}$ is invariant under the action (39). So by the Palais principle of symmetric criticality 26, the critical points of $E_{\ell}$ on $\mathfrak{M}_{c}^{r}$ are also critical points of $E_{\ell}$ on $\mathfrak{M}_{c}$;moreover these critical points clearly have the symmetry (32).

These observations show that the proof of Theorem 12 is an immediate conseguence of the following proposition: 
Proposition 14 Let the assumptions of Theorem $12 \mathrm{~V}$ be satisfied. Then, for any integer $\ell$, there exist $\bar{\delta}>0$ and a family of values of charges $c_{\delta}, \delta \in(0, \bar{\delta})$, such that $E_{\ell}$ possesses a minimizer on any $\mathfrak{M}_{c_{\delta}}^{r}$.

In order to prove Proposition 14 we shall use Theorem 8 , In this case we have

$$
\mathbf{u}=u, u \in X_{r} \text { and } E(\mathbf{u})=E_{\ell}(u), C(\mathbf{u})=C(u)=\int u^{2} d x
$$

where $E_{\ell}(u)$ is defined in (34).

Observe first that, by $\left(\overline{V_{1}}\right), E_{\ell}$ is invariant under the action $T_{k}$ of the group $G=\mathbb{Z}$ on $X_{r}$ defined by

$$
T_{k} u\left(r, x_{3}\right)=u\left(r, x_{3}+k\right), \quad k \in \mathbb{Z}
$$

Clearly $E_{\ell}$ and $C$ satisfy assumptions (EC-1), (EC-2).

In the following Lemmas we shall show that $E_{\ell}$ and $C$ satisfy also (EC-3), (EC-4) and (3).

Lemma 15 Let the assumptions of Theorem 19 be satisfied. Then $E_{\ell}$ and $C$ satisfy the coercivity assumption (EC-3).

Proof. We recall a well known inequality: there exists a constant $b_{p}>0$, such that for any $u$ in $H^{1}\left(\mathbb{R}^{N}\right)(N \geq 3)$

$$
\|u\|_{L^{p}} \leq b_{p}\|u\|_{L^{2}}^{1-N\left(\frac{1}{2}-\frac{1}{p}\right)}\|\nabla u\|_{L^{2}}^{N\left(\frac{1}{2}-\frac{1}{p}\right)} .
$$

Then

$$
\|u\|_{L^{p}}^{p} \leq b_{p}\|u\|_{L^{2}}^{p-p N\left(\frac{1}{2}-\frac{1}{p}\right)}\|\nabla u\|_{L^{2}}^{p N\left(\frac{1}{2}-\frac{1}{p}\right)} .
$$

Since $2<p<2+\frac{4}{N}$, then $p N\left(\frac{1}{2}-\frac{1}{p}\right):=q<2$. So

$$
\|u\|_{L^{p}}^{p} \leq b_{p}\|u\|_{L^{2}}^{r}\|\nabla u\|_{L^{2}}^{q}
$$

where $r=p-p N\left(\frac{1}{2}-\frac{1}{p}\right)=p-q>0$.

Then by Hölder inequality we have

$$
\begin{aligned}
\|u\|_{L^{p}}^{p} & \leq b_{p} M\|u\|_{L^{2}}^{r} \frac{1}{M}\|\nabla u\|_{L^{2}}^{q} \\
& \leq \frac{1}{\gamma^{\prime}}\left(b_{p} M\|u\|_{L^{2}}^{r}\right)^{\gamma^{\prime}}+\frac{1}{\gamma}\left(\frac{1}{M}\|\nabla u\|_{L^{2}}^{q}\right)^{\gamma} \\
& =\frac{\left(b_{p} M\right)^{\gamma^{\prime}}}{\gamma^{\prime}}\|u\|_{L^{2}}^{r \gamma^{\prime}}+\frac{1}{\gamma M^{\gamma}}\|\nabla u\|_{L^{2}}^{q \gamma} .
\end{aligned}
$$

Now chose $\gamma=\frac{2}{q}$ and $M=\left(\frac{2 c}{\gamma}\right)^{1 / \gamma}$ (where $c$ is the constant in assumption $\left.\left(\mathrm{W}_{2}\right)\right)$ so that

$$
\|u\|_{L^{p}}^{p} \leq \frac{\left(b_{p} M\right)^{\gamma^{\prime}}}{\gamma^{\prime}}\|u\|_{L^{2}}^{r \gamma^{\prime}}+\frac{1}{2 c}\|\nabla u\|_{L^{2}}^{2} .
$$


Then

$$
c\|u\|_{L^{p}}^{p} \leq a\|u\|_{L^{2}}^{2 s}+\frac{1}{2}\|\nabla u\|_{L^{2}}^{2}
$$

where

$$
a=\frac{c\left(b_{p} M\right)^{\gamma^{\prime}}}{\gamma^{\prime}} ; \quad s=\frac{r \gamma^{\prime}}{2} .
$$

So we have, taking $N=3$, and using $\left(W_{2}\right)$ and (43)

$$
\begin{aligned}
E_{\ell}(u)+a C(u)^{s} & =\frac{1}{2}\|\nabla u\|_{L^{2}}^{2}+\int V u^{2}+\ell^{2} \int \frac{u^{2}}{r^{2}}+\int W(u)+a\|u\|_{L^{2}}^{2 s} \\
& \geq \frac{1}{2}\|\nabla u\|_{L^{2}}^{2}+\int V u^{2}+\ell^{2} \int \frac{u^{2}}{r^{2}}-c \int|u|^{p}+a\|u\|_{L^{2}}^{2 s} \\
& \geq \ell^{2} \int \frac{u^{2}}{r^{2}}+\int V u^{2}
\end{aligned}
$$

Observe that, since $p>2$, we have $s>1$. So (EC-3)(i) is satisfied. Moreover it can be easily verifed that also (EC-3)(ii) is satisfied.

Now let us prove (EC-3)(iii). Let $u_{n}$ be a bounded sequence in $X_{r}$ such that $\Phi\left(u_{n}\right) \rightarrow 0$, then by (44) we have

$$
\ell^{2} \int \frac{u_{n}^{2}}{r^{2}}+\int V u_{n}^{2} \rightarrow 0
$$

So $\int u_{n}^{2} \rightarrow 0$ and $\ell^{2} \int \frac{u_{n}^{2}}{r^{2}} \rightarrow 0$. Then, in order to show that $u_{n}$ goes to 0 in $X_{r}$, it remains to prove that

$$
\left\|\nabla u_{n}\right\|_{L^{2}}^{2} \rightarrow 0 \text {. }
$$

Since $u_{n}$ is bounded in $X_{r}$, by (42) we get

$$
\int\left|u_{n}\right|^{p} \rightarrow 0
$$

Since $\Phi\left(u_{n}\right) \rightarrow 0$ and by assumption $\left(\mathrm{W}_{2}\right)$, we have

$$
0=\lim \left(E_{\ell}\left(u_{n}\right)+a C\left(u_{n}\right)^{s}\right) \geq \limsup \left(\frac{1}{2}\left\|\nabla u_{n}\right\|_{L^{2}}^{2}+D_{n}\right)
$$

where

$$
D_{n}=\ell^{2} \int \frac{u_{n}^{2}}{r^{2}}+\int V u_{n}^{2}+a \int u_{n}^{2}-c \int\left|u_{n}\right|^{p} .
$$

By (45) and (47) we get $D_{n} \rightarrow 0$. So by (48) we deduce (46).

Lemma 16 Let the assumptions of theorem 12 be satisfied. Then $E_{\ell}$ and $C$ satisfy the splitting property (EC-4). 
Proof. Consider any sequence

$$
u_{n}=u+w_{n} \in X_{r}
$$

where $w_{n}$ converges weakly to 0 . We set

$$
E_{\ell}(v)=A(v, v)+K(v)
$$

where

$$
A(v, v)=\int_{\mathbb{R}^{3}}\left[\frac{1}{2}|\nabla v|^{2}+\left(\frac{1}{2} \frac{\ell^{2}}{r^{2}}+V(x)\right) v^{2}\right]
$$

and

$$
K(v)=\int W(v) d x .
$$

Since $C(v)=\int v^{2}$ and $A(v, v)$ are quadratic, by remark 6, we have only to show that $K(v)$ satisfies the splitting property. For any measurable $A \subset \mathbb{R}^{3}$ and any $\nu \in X_{r}$, we set

$$
K_{A}(v)=\int_{A} W(v) d x .
$$

Choose $\varepsilon>0$ and $R=R(\varepsilon)>0$ such that

$$
\left|K_{B_{R}^{c}}(u)\right|<\varepsilon
$$

where

$$
B_{R}^{c}=\mathbb{R}^{N}-B_{R} \text { and } B_{R}=\left\{x \in \mathbb{R}^{N}:|x|<R\right\} .
$$

Since $w_{n} \rightarrow 0$ weakly in $H^{1}\left(\mathbb{R}^{3}\right)$, by usual compactness arguments, we have that

$$
K_{B_{R}}\left(w_{n}\right) \rightarrow 0 \text { and } K_{B_{R}}\left(u+w_{n}\right) \rightarrow K_{B_{R}}(u) .
$$

Then, by (50) and (51), we have

$$
\begin{aligned}
& \lim _{n \rightarrow \infty}\left|K\left(u+w_{n}\right)-K(u)-K\left(w_{n}\right)\right| \\
& =\lim _{n \rightarrow \infty}\left|K_{B_{R}^{c}}\left(u+w_{n}\right)+K_{B_{R}}\left(u+w_{n}\right)-K_{B_{R}^{c}}(u)-K_{B_{R}}(u)-K_{B_{R}^{c}}\left(w_{n}\right)-K_{B_{R}}\left(w_{n}\right)\right| \\
& =\lim _{n \rightarrow \infty}\left|K_{B_{R}^{c}}\left(u+w_{n}\right)-K_{B_{R}^{c}}(u)-K_{B_{R}^{c}}\left(w_{n}\right)\right| \\
& \leq \lim _{n \rightarrow \infty}\left|K_{B_{R}^{c}}\left(u+w_{n}\right)-K_{B_{R}^{c}}\left(w_{n}\right)\right|+\varepsilon .
\end{aligned}
$$

Now, by the intermediate value theorem, there exists $\zeta_{n} \in(0,1)$ such that for $z_{n}=\zeta_{n} u+\left(1-\zeta_{n}\right) w_{n}$, we have that

$$
\left|K_{B_{R}^{c}}\left(u+w_{n}\right)-K_{B_{R}^{c}}\left(w_{n}\right)\right|=\left|\left\langle K_{B_{R}^{c}}^{\prime}\left(z_{n}\right), u\right\rangle\right|
$$




$$
\begin{aligned}
& \leq \int_{B_{R}^{c}}\left|W^{\prime}\left(z_{n}\right) u\right| \leq\left(\text { by }\left(\overline{W_{1}}\right)\right) \\
& \leq \int_{B_{R}^{c}} c_{1}\left|z_{n}\right|^{r-1}|u|+c_{2}\left|z_{n}\right|^{q-1}|u| \\
& \leq c_{1}\left\|z_{n}\right\|_{L^{r}\left(B_{R}^{c}\right)}^{r-1}\|u\|_{L^{r}\left(B_{R}^{c}\right)}+c_{2}\left\|z_{n}\right\|_{L^{q}\left(B_{R}^{c}\right)}^{q-1}\|u\|_{L^{q}\left(B_{R}^{c}\right)}
\end{aligned}
$$

(if $R$ is large enough)

$$
\leq c_{3}\left(\left\|z_{n}\right\|_{L^{r}\left(B_{R}^{c}\right)}^{r-1}+\left\|z_{n}\right\|_{L^{q}\left(B_{R}^{c}\right)}^{q-1}\right) \varepsilon
$$

So we have

$$
\left|K_{B_{R}^{c}}\left(u+w_{n}\right)-K_{B_{R}^{c}}\left(w_{n}\right)\right| \leq c_{3}\left(\left\|z_{n}\right\|_{L^{r}\left(B_{R}^{c}\right)}^{r-1}+\left\|z_{n}\right\|_{L^{q}\left(B_{R}^{c}\right)}^{q-1}\right) \varepsilon
$$

Since $z_{n}$ is bounded in $H^{1}\left(\mathbb{R}^{3}\right)$, the sequences $\left\|z_{n}\right\|_{L^{r}\left(B_{R}^{c}\right)}^{r-1}$ and $\left\|z_{n}\right\|_{L^{q}\left(B_{R}^{c}\right)}^{q-1}$ are bounded. Then, by (52) and (58), we easily get

$$
\lim _{n \rightarrow \infty}\left|K\left(u+w_{n}\right)-K(u)-K\left(w_{n}\right)\right| \leq \varepsilon+M \cdot \varepsilon .
$$

where $M$ is a suitable constant.

Since $\varepsilon$ is arbitrary, from (59) we get

$$
\lim _{n \rightarrow \infty}\left|K\left(u+w_{n}\right)-K(u)-K\left(w_{n}\right)\right|=0
$$

Now in order to prove that assumption (3) is satisfied some work is necessary. Set

$$
\Lambda(u)=\frac{E_{\ell}(u)}{C(u)} .
$$

First of all we have:

Lemma 17 If the assumptions of Theorem 12 are satisfied, then for $6>t>2$, we have

$$
\liminf _{u \in X_{r},\|u\|_{L^{t}} \rightarrow 0} \Lambda(u)=\inf _{u \in X_{r}\|u\|_{L t}=1} \frac{\frac{1}{2} \int\left(|\nabla u|^{2}+\frac{\ell^{2} u^{2}}{r^{2}}\right) d x+\int V u^{2}}{\int u^{2}} .
$$

Proof. Clearly

$$
\begin{gathered}
\liminf _{u \in X_{r},\|u\|_{L^{t} \rightarrow 0}} \Lambda(u)=\liminf _{u \in X_{r},\|u\|_{L^{t}}=1, \varepsilon \rightarrow 0} \frac{E(\varepsilon u)}{C(\varepsilon u)} \\
=\inf _{u \in X_{r},\|u\|_{L^{t}}=1}\left(\frac{\frac{1}{2} \int\left(|\nabla u|^{2}+\frac{\ell^{2} u^{2}}{r^{2}}\right) d x+\int V u^{2}}{\int u^{2}}\right)+\liminf _{u \in X_{r},\|u\|_{L^{t}}=1, \varepsilon \rightarrow 0} \frac{\int W(\varepsilon u)}{\varepsilon^{2} \int u^{2}} .
\end{gathered}
$$


So the proof of Lemma will be achieved if we show that

$$
\liminf _{u \in X_{r},\|u\|_{L^{t}}=1, \varepsilon \rightarrow 0} \frac{\int W(\varepsilon u)}{\varepsilon^{2} \int u^{2}}=0 .
$$

By assumptions $\left(W_{1}\right)$ and $\left(W_{2}\right)$ we have

$$
-c s^{p} \leq W(s) \leq \bar{c}\left(s^{q}+s^{r}\right)
$$

where $c, \bar{c}$ are positive constants and $q, r$ in $(2,6)$.

Then by (61) we have

$$
-c A \varepsilon^{p-2} \leq \inf _{\|u\|_{L^{t}}=1} \frac{\int W(\varepsilon u)}{\varepsilon^{2} \int u^{2}} \leq \bar{c} B\left(\varepsilon^{q-2}+\varepsilon^{r-2}\right)
$$

where

$$
A=\inf _{u \in X_{r}\|u\|_{L^{t}}=1} \frac{\int|u|^{p}}{\int u^{2}}, B=\inf _{u \in X_{r}\|u\|_{L^{t}}=1} \frac{\int\left(|u|^{q}+|u|^{r}\right)}{\int u^{2}} .
$$

By (62) we easily get (60).

Now consider the following action $T_{k}$ of the group $G=\mathbb{Z}$ on $X_{r}$ :

$$
\text { for all } u \in X_{r}, k \in \mathbb{Z} T_{k} u\left(x_{1}, x_{2}, x_{3}\right)=u\left(x_{1}, x_{2}, x_{3}+k\right)
$$

The following proposition holds

Lemma 18 If $2<t<6$, the norm $\|u\|_{L^{t}}$ satisfies the property (5), namely

$$
\left\{u_{n} \text { is a vanishing sequence }\right\} \Rightarrow\left\|u_{n}\right\|_{L^{t}} \rightarrow 0 .
$$

Proof. Let $u_{n}$ be a G-vanishing sequence in $X_{r}$ and, arguing by contradiction, assume that $\left\|u_{n}\right\|_{L^{t}}$ does not converge to 0 . Then, up to a subsequence,

$$
\left\|u_{n}\right\|_{L^{t}} \geq a>0 .
$$

Since $u_{n}$ is bounded in $X_{r}$, we have that for a suitable constant $M>0$

$$
\left\|u_{n}\right\|_{H^{1}}^{2} \leq M
$$

Now we set

$$
Q_{i}=\left\{\left(x_{1}, x_{2}, x_{3}\right): i \leq x_{3}<i+1\right\}, i \text { integer. }
$$

Clearly

$$
\mathbb{R}^{3}=\bigcup_{i \in \mathbb{Z}} Q_{i}
$$


Let $C$ denote the constant for the Sobolev embedding $H^{1}\left(Q_{i}\right) \subset L^{t}\left(Q_{i}\right)$, then, by (63) and (64), we get the following

$$
\begin{aligned}
0 & <a^{t} \leq \int\left|u_{n}\right|^{t}=\sum_{i} \int_{Q_{i}}\left|u_{n}\right|^{t}=\sum_{i}\left\|u_{n}\right\|_{L^{t}\left(Q_{i}\right)}^{t-2}\left\|u_{n}\right\|_{L^{t}\left(Q_{i}\right)}^{2} \\
& \leq\left(\sup _{i}\left\|u_{n}\right\|_{L^{t}\left(Q_{i}\right)}^{t-2}\right) \cdot \sum_{i}\left\|u_{n}\right\|_{L^{t}\left(Q_{i}\right)}^{2} \\
& \leq C\left(\sup _{i}\left\|u_{n}\right\|_{L^{t}\left(Q_{i}\right)}^{t-2}\right) \cdot \sum_{i}\left\|u_{n}\right\|_{H^{1}\left(Q_{i}\right)}^{2} \\
& =C\left(\sup _{i}\left\|u_{n}\right\|_{L^{t}\left(Q_{i}\right)}^{t-2}\right)\left\|\mathbf{u}_{n}\right\|_{H^{1}}^{2} \leq C M\left(\sup _{i}\left\|u_{n}\right\|_{L^{t}\left(Q_{i}\right)}^{t-2}\right) .
\end{aligned}
$$

Then

$$
\left(\sup _{i}\left\|u_{n}\right\|_{L^{t}\left(Q_{i}\right)}\right) \geq\left(\frac{a^{t}}{C M}\right)^{1 /(t-2)} .
$$

So, for any $n$, there exists an integer $i_{n}$ such that

$$
\left\|u_{n}\right\|_{L^{t}\left(Q_{i_{n}}\right)} \geq \alpha>0 .
$$

Then

$$
\left\|T_{i_{n}} u_{n}\right\|_{L^{t}\left(Q_{0}\right)}=\left\|u_{n}\right\|_{L^{t}\left(Q_{i_{n}}\right)} \geq \alpha>0 .
$$

Since $u_{n}$ and then $T_{i_{n}} u_{n}$ is bounded in $X_{r}$, we have, passing eventually to a subsequence, that

$$
T_{i_{n}} u_{n} \rightarrow u_{0} \text { weakly in } X_{r} .
$$

Clearly, if we show that $u_{0} \neq 0$, we get a contradiction with the assumption that $u_{n}$ is nonvanishing.

Now, let $\varphi=\varphi\left(x_{3}\right)$ be a nonnegative, $C^{\infty}$-function whose value is 1 for $0<x_{3}<1$ and 0 for $\left|x_{3}\right|>2$. Then the sequence $\varphi T_{i_{n}} u_{n}$ is bounded in $H_{0}^{1}\left(\mathbb{R}^{2} \times(-2,2)\right)$, moreover $\varphi T_{i_{n}} u_{n}$ is invariant under the action (39). Then, using the compactness result proved in 21, we have

$$
\varphi T_{i_{n}} u_{n} \rightarrow \chi \text { strongly in } L^{t}\left(\mathbb{R}^{2} \times(-2,2)\right) .
$$

On the other hand

$$
\varphi T_{i_{n}} u_{n} \rightarrow \varphi u_{0} \text { a.e. }
$$

Then

$$
\varphi T_{i_{n}} u_{n} \rightarrow \varphi u_{0} \text { strongly in } L^{t}\left(\mathbb{R}^{2} \times(-2,2)\right) .
$$

Moreover

$$
\left\|\varphi T_{i_{n}} u_{n}\right\|_{L^{3}\left(\mathbb{R}^{2} \times(-2,2)\right)} \geq\left\|\varphi T_{i_{n}} u_{n}\right\|_{L^{t}\left(Q_{0}\right)}=\left\|u_{n}\right\|_{L^{t}\left(Q_{i_{n}}\right)} \geq \alpha>0 .
$$

Then by (67) and (68)

$$
\left\|\varphi u_{0}\right\|_{L^{3}\left(\mathbb{R}^{2} \times(-2,2)\right)} \geq \alpha>0 .
$$


Thus we have that $u_{0} \neq 0$.

Finally it remains to show that also assumption (3) is satisfied.

Lemma 19 Let the assumptions of Theorem 12 be satisfied, then

$$
\inf _{u \in X_{r}} \Lambda(u)<\Lambda_{0}
$$

Proof. We shall show that there exists $u \in X_{r}$ such that $\Lambda(u)<\Lambda_{0}$. The construction of such $u$ needs some work since we require that $u$ belongs to $X_{r}$, namely we require that $u$ is invariant under the $S^{1}$ action (39) and it is 0 near the $x_{3}$ axis, so that $\int \frac{u^{2}}{r^{2}}$ converges.

For $0<\mu<\lambda$ we set:

$$
T_{\lambda, \mu}=\left\{\left(r, x_{3}\right):(r-\lambda)^{2}+x_{3}^{2} \leq \mu^{2}\right\}
$$

and, for $\lambda>2$, we consider a smooth function $u_{\lambda}$ with cylindrical symmetry such that

$$
u_{\lambda}\left(r, x_{3}\right)=\left\{\begin{array}{ccc}
s_{0} & \text { if } \quad\left(r, x_{3}\right) \in T_{\lambda, \lambda / 2} \\
0 & \text { if } \quad\left(r, x_{3}\right) \notin T_{\lambda, \lambda / 2+1}
\end{array}\right.
$$

where $s_{0}$ is such that $\frac{W\left(s_{0}\right)}{s_{0}^{2}}<\inf V-\sup V\left(\right.$ see $\left.\left(\mathrm{W}_{3}\right)\right)$. Moreover we may assume that

$$
\left|\nabla u_{\lambda}\left(r, x_{3}\right)\right| \leq 2 \text { for }\left(r, x_{3}\right) \in T_{\lambda, \lambda / 2+1} \backslash T_{\lambda, \lambda / 2} .
$$

We have

$$
\Lambda\left(u_{\lambda}\right)=\frac{\int\left[\left|\nabla u_{\lambda}\right|^{2}+\frac{\ell^{2} u_{\lambda}^{2}}{r^{2}}+2 V u^{2}\right] d x}{2 \int u_{\lambda}^{2}}+\frac{\int W\left(u_{\lambda}\right) d x}{\int u_{\lambda}^{2}} .
$$

By (70) and (69) a direct computation shows that

$$
\begin{gathered}
\int\left|\nabla u_{\lambda}\right|^{2} \leq 4 \operatorname{meas}\left(T_{\lambda, \lambda / 2+1} \backslash T_{\lambda, \lambda / 2}\right) \leq c_{1} \lambda^{2} \\
\int \frac{u_{\lambda}^{2}}{r^{2}} \leq \frac{c_{2}}{\lambda^{2}} \operatorname{meas}\left(T_{\lambda, \lambda / 2+1}\right) \leq c_{3} \lambda \\
\int u_{\lambda}^{2} \geq c_{4} \text { meas }\left(T_{\lambda, \lambda / 2}\right) \geq c_{5} \lambda^{3}
\end{gathered}
$$

where $c_{1}, . ., c_{5}$ are positive constants. So that

$$
\frac{\int\left[\left|\nabla u_{\lambda}\right|^{2}+\frac{\ell^{2} u_{\lambda}^{2}}{r^{2}}+2 V u_{\lambda}^{2}\right] d x}{2 \int u_{\lambda}^{2}} \leq \sup V+O\left(\frac{1}{\lambda}\right) .
$$


Now

$$
\int_{T_{\lambda, \lambda / 2+1} \backslash T_{\lambda, \lambda / 2}}\left|W\left(u_{\lambda}\right)\right| \leq c_{6} \operatorname{meas}\left(T_{\lambda, \lambda / 2+1} \backslash T_{\lambda, \lambda / 2}\right) \leq c_{6} \lambda^{2}
$$

Then

$$
\begin{aligned}
\int W\left(u_{\lambda}\right) d x & =W\left(s_{0}\right) \operatorname{meas}\left(T_{\lambda, \lambda / 2}\right)+\int_{T_{\lambda, \lambda / 2+1} \backslash T_{\lambda, \lambda / 2}} W\left(u_{\lambda}\right) \\
& \leq W\left(s_{0}\right) \text { meas }\left(T_{\lambda, \lambda / 2}\right)+\int_{T_{\lambda, \lambda / 2+1} \backslash T_{\lambda, \lambda / 2}}\left|W\left(u_{\lambda}\right)\right| \\
& \leq W\left(s_{0}\right) \text { meas }\left(T_{\lambda, \lambda / 2}\right)+c_{6} \lambda^{2} .
\end{aligned}
$$

So

$$
\begin{gathered}
\frac{\int W\left(u_{\lambda}\right) d x}{\int u_{\lambda}^{2}} \leq \frac{W\left(s_{0}\right) \operatorname{meas}\left(T_{\lambda, \lambda / 2}\right)+c_{6} \lambda^{2}}{\int u_{\lambda}^{2}} \leq \\
\frac{W\left(s_{0}\right) \operatorname{meas}\left(T_{\lambda, \lambda / 2}\right)}{\int u_{\lambda}^{2}}+\frac{c_{6} \lambda^{2}}{\int u_{\lambda}^{2}} .
\end{gathered}
$$

Now, since $W\left(s_{0}\right)<0$, we have

$$
\frac{W\left(s_{0}\right) \operatorname{meas}\left(T_{\lambda, \lambda / 2}\right)}{\int u_{\lambda}^{2}} \leq \frac{W\left(s_{0}\right) \operatorname{meas}\left(T_{\lambda, \lambda / 2}\right)}{s_{0}^{2} \operatorname{meas}\left(T_{\lambda, \lambda / 2+1}\right)}=\frac{W\left(s_{0}\right)}{s_{0}^{2}}\left(\frac{\lambda}{\lambda+2}\right)^{2} .
$$

Then by (74), (76) and (77) we have

$$
\frac{\int W\left(u_{\lambda}\right) d x}{\int u_{\lambda}^{2}} \leq \frac{W\left(s_{0}\right)}{s_{0}^{2}}\left(\frac{\lambda}{\lambda+2}\right)^{2}+\frac{c_{7}}{\lambda} .
$$

By (71), (75) and (78) we get

$$
\begin{aligned}
\Lambda\left(u_{\lambda}\right) & \leq \sup V+\frac{W\left(s_{0}\right)}{s_{0}^{2}}\left(\frac{\lambda}{\lambda+2}\right)^{2}+O\left(\frac{1}{\lambda}\right) . \\
& \sup V-\inf V+\inf V+\frac{W\left(s_{0}\right)}{s_{0}^{2}}\left(\frac{\lambda}{\lambda+2}\right)^{2}+O\left(\frac{1}{\lambda}\right) .
\end{aligned}
$$

By lemma 17 we have

$$
\liminf _{\mathbf{u} \in X_{r},\|\mathbf{u}\|_{L^{t}} \rightarrow 0} \Lambda(\mathbf{u}) \geq \inf V
$$

then

$$
\Lambda\left(u_{\lambda}\right) \leq \sup V-\inf V+\liminf _{\mathbf{u} \in X_{r},\|\mathbf{u}\|_{L^{t}} \rightarrow 0} \Lambda(\mathbf{u})+\frac{W\left(s_{0}\right)}{s_{0}^{2}}\left(\frac{\lambda}{\lambda+2}\right)^{2}+O\left(\frac{1}{\lambda}\right) .
$$

By assumption $\left(\mathrm{W}_{3}\right)$ for $\lambda$ large we have

$$
\sup V-\inf V+\frac{W\left(s_{0}\right)}{s_{0}^{2}}\left(\frac{\lambda}{\lambda+2}\right)^{2}+O\left(\frac{1}{\lambda}\right)<0
$$


By (79) and (80) we get that for $\lambda$ large

$$
\Lambda\left(u_{\lambda}\right)<\liminf _{\mathbf{u} \in X_{r},\|u\|_{L^{t} \rightarrow 0}} \Lambda(u) .
$$

On the other hand, since by Lemma 18\|\|$_{L^{t}}$ satisfies the property (5), we have by (6) that

$$
\liminf _{u \in X_{r},\|u\|_{L^{t}} \rightarrow 0} \Lambda(u) \leq \Lambda_{0} .
$$

Clearly (81) and (82) imply that assumption (3) is satisfied.

\section{Existence of hylomorphic solitons}

\subsection{An abstract definition of solitary waves and solitons}

Solitons are particular states of a dynamical system described by one or more partial differential equations. Thus we assume that the states of this system are described by one or more fields which mathematically are represented by functions

$$
\mathbf{u}: \mathbb{R}^{N} \rightarrow V
$$

where $V$ is a vector space with norm $|\cdot|_{V}$ and which is called the internal parameters space. We assume the system to be deterministic; this means that it can be described as a dynamical system $(X, \gamma)$ where $X$ is the set of the states and $\gamma: \mathbb{R} \times X \rightarrow X$ is the time evolution map. If $\mathbf{u}_{0}(x) \in X$, the evolution of the system will be described by the function

$$
\mathbf{u}(t, x):=\gamma_{t} \mathbf{u}_{0}(x) .
$$

We assume that the states of $X$ have "finite energy" so that they decay at $\infty$ sufficiently fast. Roughly speaking, the solitons are "bump" solutions characterized by some form of stability.

To define them at this level of abstractness, we need to recall some well known notions in the theory of dynamical systems.

Definition $20 A$ set $\Gamma \subset X$ is called invariant if $\forall \mathbf{u} \in \Gamma, \forall t \in \mathbb{R}, \gamma_{t} \mathbf{u} \in \Gamma$.

Definition 21 Let $(X, d)$ be a metric space and let $(X, \gamma)$ be a dynamical system. An invariant set $\Gamma \subset X$ is called stable, if $\forall \varepsilon>0, \exists \delta>0, \forall \mathbf{u} \in X$,

$$
d(\mathbf{u}, \Gamma) \leq \delta,
$$

implies that

$$
\forall t \geq 0, d\left(\gamma_{t} \mathbf{u}, \Gamma\right) \leq \varepsilon
$$


Let $G$ be a subgroup of $\left(\mathbb{R}^{N},+\right)$ and consider the following action $T_{z}$ of $G$ on $X$ : for all $z \in G$ and $\mathbf{u} \in X$

$$
T_{z} \mathbf{u}(x)=\mathbf{u}(x+z) .
$$

Now we are ready to give the definition of soliton:

Definition 22 A state $\mathbf{u}(x) \in X$ is called soliton if there is an invariant set $\Gamma$ such that

- (i) $\forall t, \gamma_{t} \mathbf{u}(x) \in \Gamma$,

- (ii) $\Gamma$ is stable,

- (iii) $\Gamma$ is G-compact (Def. [3)

Remark 23 The above definition needs some explanation. For simplicity, we assume that $\Gamma$ is a manifold (actually, in many concrete models, this is the generic case). Then (iii) implies that $\Gamma$ is finite dimensional. Since $\Gamma$ is invariant, $\mathbf{u}_{0} \in \Gamma \Rightarrow \gamma_{t} \mathbf{u}_{0} \in \Gamma$ for every time. Thus, since $\Gamma$ is finite dimensional, the evolution of $\mathbf{u}_{0}$ is described by a finite number of parameters. The dynamical system $(\Gamma, \gamma)$ behaves as a point in a finite dimensional phase space. By the stability of $\Gamma$, a small perturbation of $\mathbf{u}_{0}$ remains close to $\Gamma$. However, in this case, its evolution depends on an infinite number of parameters. Thus, this system appears as a finite dimensional system with a small perturbation.

Remark 24 The type of stability described above is called orbital stability in the literature relative to the nonlinear Schrödinger and Klein-Gordon equations.

\subsection{An existence result for hylomorphic solitons}

We now assume that the dynamical system $(X, \gamma)$ has two constants of motion. These constants can be considered as functionals on $X$. One of them will be called energy and it will be denoted by $E$; the other will be called hylenic charge and it will be denoted by $C$.

At this level of abstractness, the names energy and hylenic charge are conventional but $E$ and $C$ satisfy different assumptions; see assumption (EC-3) in section 2.1. In our applications to PDE's, $E$ will be the usual energy. The name hylenic charge has been introduced in [9, 4] and [5].

The presence of $E$ and $C$ allows to give the following definition of hylomorphic soliton.

Definition $25 A$ soliton $\mathbf{u}_{0} \in X$ is called hylomorphic if $\Gamma$ (as in Def. 22) has the following structure

$$
\Gamma=\Gamma\left(e_{0}, c_{0}\right)=\left\{\mathbf{u} \in X \mid E(\mathbf{u})=e_{0}, C(\mathbf{u})=c_{0}\right\}
$$

where

$$
e_{0}=\min \left\{E(\mathbf{u}) \mid C(\mathbf{u})=c_{0}\right\}
$$

for some $c_{0} \in \mathbb{R}$. 
Notice that, by (85), we have that a hylomorphic soliton $\mathbf{u}_{0}$ satisfies the following nonlinear eigenvalue problem:

$$
E^{\prime}\left(\mathbf{u}_{0}\right)=\lambda C^{\prime}\left(\mathbf{u}_{0}\right) .
$$

Clearly, for a given $c_{0}$ the minimum $e_{0}$ in (85) might not exist; moreover, even if the minimum exists, it is possible that $\Gamma$ does not satisfy (ii) or (iii) of def. 22 ,

The following theorem holds

Theorem 26 Assume that the dynamical system $(X, \gamma)$ satisfies $(E C-1), \ldots,(E C$ 4) and (3). Moreover assume that

$$
E \text { and } C \text { are two constants of motion. }
$$

Then there exists $\bar{\delta}>0$ such that the dynamical system $(X, \gamma)$ admits a family $\mathbf{u}_{\delta}(\delta \in(0, \bar{\delta}))$ of hylomorphic solitons.

The proof of this theorem will be given in the next section.

\subsection{A stability result}

In order to prove Theorem 26] it is sufficient to show that the minimizers in Th. 8 provide solitons, so we have to prove that the set $\Gamma_{c_{\delta}}$ is stable. To do this, we need the (well known) Liapunov theorem in following form:

Theorem 27 Let $\Gamma$ be an invariant set and assume that there exists a differentiable function $V$ (called a Liapunov function) such that

- (a) $V(\mathbf{u}) \geq 0$ and $V(\mathbf{u})=0 \Leftrightarrow u \in \Gamma$

- (b) $\partial_{t} V\left(\gamma_{t}(\mathbf{u})\right) \leq 0$

- (c) $V\left(\mathbf{u}_{n}\right) \rightarrow 0 \Leftrightarrow d\left(\mathbf{u}_{n}, \Gamma\right) \rightarrow 0$.

Then $\Gamma$ is stable.

Proof. For completeness, we give a proof of this well known result. Arguing by contradiction, assume that $\Gamma$, satisfying the assumptions of Th. 27, is not stable. Then there exists $\varepsilon>0$ and sequences $\mathbf{u}_{n} \in X$ and $t_{n}>0$ such that

$$
d\left(\mathbf{u}_{n}, \Gamma\right) \rightarrow 0 \text { and } d\left(\gamma_{t_{n}}\left(\mathbf{u}_{n}\right), \Gamma\right)>\varepsilon .
$$

Then we have

$$
d\left(\mathbf{u}_{n}, \Gamma\right) \rightarrow 0 \Longrightarrow V\left(\mathbf{u}_{n}\right) \rightarrow 0 \Longrightarrow V\left(\gamma_{t_{n}}\left(\mathbf{u}_{n}\right)\right) \rightarrow 0 \Longrightarrow d\left(\gamma_{t_{n}}\left(\mathbf{u}_{n}\right), \Gamma\right) \rightarrow 0
$$

where the first and the third implications are consequence of property (c). The second implication follows from property (b). Clearly, this fact contradicts (86). 
Theorem 28 Assume (EC-1) and (EC-2). For $\mathbf{u} \in X$ and $e_{0}, c_{0} \in \mathbb{R}$, we set

$$
V(\mathbf{u})=\left(E(\mathbf{u})-e_{0}\right)^{2}+\left(C(\mathbf{u})-c_{0}\right)^{2} .
$$

If $V$ is G-compact (see Def. 杘) and

$$
\Gamma=\left\{\mathbf{u} \in X: E(\mathbf{u})=e_{0}, C(\mathbf{u})=c_{0}\right\} \neq \varnothing,
$$

then every $\mathbf{u} \in \Gamma$ is a soliton.

Proof: We have to prove that $\Gamma$ in (88) satisfies (i),(ii) and (iii) of Def. 22 The property (iii), namely the fact that $\Gamma$ is G-compact, is a trivial consequence of the fact that $\Gamma$ is the set of minimizers of a G-compact functional $V$ (see definitions 3 and (4). The invariance property (i) is clearly satisfied since $E$ and $C$ are constants of the motion. It remains to prove (ii), namely that $\Gamma$ is stable. To this end we shall use Th. [27. So we need to show that $V(\mathbf{u})$ satisfies (a), (b) and (c). Statements (a) and (b) are trivial. Now we prove (c). First we show the implication $\Rightarrow$. Let $\mathbf{u}_{n}$ be a sequence such that $V\left(\mathbf{u}_{n}\right) \rightarrow 0$. By contradiction we assume that $d\left(\mathbf{u}_{n}, \Gamma\right) \nrightarrow 0$, namely that there is a subsequence $\mathbf{u}_{n}^{\prime}$ such that

$$
d\left(\mathbf{u}_{n}^{\prime}, \Gamma\right) \geq a>0 .
$$

Since $V\left(\mathbf{u}_{n}\right) \rightarrow 0$ also $V\left(\mathbf{u}_{n}^{\prime}\right) \rightarrow 0$, and, since $V$ is $G$ compact, there exists a sequence $g_{n}$ in $G$ such that, for a subsequence $\mathbf{u}_{n}^{\prime \prime}$, we have $g_{n} \mathbf{u}_{n}^{\prime \prime} \rightarrow \mathbf{u}_{0}$. Then

$$
d\left(\mathbf{u}_{n}^{\prime \prime}, \Gamma\right)=d\left(g_{n} \mathbf{u}_{n}^{\prime \prime}, \Gamma\right) \leq d\left(g_{n} \mathbf{u}_{n}^{\prime \prime}, \mathbf{u}_{0}\right) \rightarrow 0
$$

and this contradicts (89).

Now we prove the other implication $\Leftarrow$. Let $\mathbf{u}_{n}$ be a sequence such that $d\left(\mathbf{u}_{n}, \Gamma\right) \rightarrow 0$, then there exists $\mathbf{v}_{n} \in \Gamma$ s.t.

$$
d\left(\mathbf{u}_{n}, \Gamma\right) \geq d\left(\mathbf{u}_{n}, \mathbf{v}_{n}\right)-\frac{1}{n} .
$$

Since $V$ is G-compact, also $\Gamma$ is G-compact; so, for a suitable sequence $g_{n}$, we have $g_{n} \mathbf{v}_{n} \rightarrow \overline{\mathbf{w}} \in \Gamma$. We get the conclusion if we show that $V\left(\mathbf{u}_{n}\right) \rightarrow 0$. We have by (90), that $d\left(\mathbf{u}_{n}, \mathbf{v}_{n}\right) \rightarrow 0$ and hence $d\left(g_{n} \mathbf{u}_{n}, g_{n} \mathbf{v}_{n}\right) \rightarrow 0$ and so, since $g_{n} \mathbf{v}_{n} \rightarrow \overline{\mathbf{w}}$, we have $g_{n} \mathbf{u}_{n} \rightarrow \overline{\mathbf{w}} \in \Gamma$. Therefore, by the continuity of $V$ and since $\overline{\mathbf{w}} \in \Gamma$, we have $V\left(g_{n} \mathbf{u}_{n}\right) \rightarrow V(\overline{\mathbf{w}})=0$ and we can conclude that $V\left(\mathbf{u}_{n}\right) \rightarrow 0$.

In the cases in which we are interested, $X$ is an infinite dimensional manifold; then if you choose generic $e_{0}$ and $c_{0}, V$ is not $G$-compact since the set $\Gamma=$ $\left\{\mathbf{u} \in X: E(\mathbf{u})=e_{0}, C(\mathbf{u})=c_{0}\right\}$ has codimension 2. However, Th. (8) allows to determine $e_{0}$ and $c_{0}$ in such a way that $V$ is $G$-compact and hence to prove the existence of solitons by using Theorem 28

Proof of Th. 26, In order to prove Th. 26, we will use Th. 28 with $e_{0}=e_{\delta}$ and $c_{0}=c_{\delta}$ where $e_{\delta}$ and $c_{\delta}$ are given by Th. 8 , 
We set

$$
V(\mathbf{u})=\left(E(\mathbf{u})-e_{\delta}\right)^{2}+\left(C(\mathbf{u})-c_{\delta}\right)^{2} .
$$

We show that $V$ is $G$-compact: let $\mathbf{w}_{n}$ be a minimizing sequence for $V$, then $V\left(\mathbf{w}_{n}\right) \rightarrow 0$ and consequently $E\left(\mathbf{w}_{n}\right) \rightarrow e_{\delta}$ and $C\left(\mathbf{w}_{n}\right) \rightarrow c_{\delta}$. Let $J_{\delta}$ be as in Theorem 8, Now, since

$$
\inf J_{\delta}=\frac{e_{\delta}}{c_{\delta}}+\delta\left[e_{\delta}+a c_{\delta}^{s}\right],
$$

we have that $\mathbf{w}_{n}$ is a minimizing sequence also for $J_{\delta}$. Then, since $J_{\delta}$ is $G$ compact, we get

$$
\mathbf{w}_{n} \text { is } G \text {-compact. }
$$

So we conclude that $V$ is $G$-compact and hence the conclusion follows by using Theorem 28

\section{Existence of solitons for NSE with periodic potential}

In this section we shall study the existence of hylomorphic solitons on lattice for the Schrödinger equation (NSE) in $\mathbb{R}^{N}$.

The existence of solitons for (NSE) is an old problem and there are many results in the case $V=0$ ([20, [19, 6] and the references in [9]).

Here we assume that $V$ is a lattice potential, namely we assume that the potential $V$ satisfies the periodicity condition.

$$
V(x)=V(x+A z) \text { for all } x \in \mathbb{R}^{N} \text { and } z \in \mathbb{Z}^{N}
$$

where $A$ is a $N \times N$ invertible matrix.

Here we look for solitons and do not require they to be vortices, so the energy corresponds to the expression (34) with $\ell=0$, namely

$$
E(u)=E_{0}(u)=\int_{\mathbb{R}^{3}}\left[\frac{1}{2}|\nabla u|^{2}+V(x) u^{2}+W(u)\right] d x, u \in X .
$$

As before the charge is

$$
C(u)=\int u^{2} d x
$$

In this case $X$ is the ordinary $H^{1}\left(\mathbb{R}^{N}\right)$ Sobolev space. We shall consider the following action of the group $G=\mathbb{Z}^{N}$ on $X$ :

$$
\text { for all } z \in \mathbb{Z}^{N} \text { and } u \in X: T_{z} u(x)=u(x+A z) \text {. }
$$

Clearly the charge $C$ is $G$-invariant and, since $V$ satisfies $\left(V 1^{\prime}\right)$, also the energy $E$ is invariant under this group action. The following Theorem holds: 
Theorem 29 Let $W$ and $V$ satisfy assumptions $\left(W_{0}\right), \ldots,\left(W_{3}\right)$ and $\left(V_{0}\right),\left(V_{1}^{\prime}\right)$. Then there exists $\bar{\delta}>0$ such that the dynamical system described by the Schrödinger equation (NSE) has a family $u_{\delta}(\delta \in(0, \bar{\delta}))$ of hylomorphic solitons.

The proof of this theorem is based on the abstract theorem 26, In this case the energy is given by (93). We need to show that assumptions $\left(W_{0}\right), \ldots,\left(W_{3}\right)$ and $\left(V_{0}\right),\left(V_{1}^{\prime}\right)$ permit to show that assumptions (EC-1), ...,(EC-4) and (3) of theorem 26 are satisfied.

(EC-1) and (EC-2) are trivially verified. The proof of the other assumptions follows the same lines of the proof of Th. 12 as we can see in the following lemmas:

Lemma $30 E$ and $C$ satisfy the coercivity assumption (EC-3).

Proof. The proof is the same of that of lemma 15 with $\ell=0$.

Lemma $31 E$ and $C$ satisfy the splitting property (EC-4).

Proof. The proof is the same of that of lemma 16 with $\ell=0$.

Lemma 32 If $2<t<\frac{2 N}{N-2}, N \geq 3$, the norm $\|u\|_{L^{t}}$ satisfies the property (5), namely

$$
\left\{\mathbf{u}_{n} \text { is a vanishing sequence }\right\} \Rightarrow\left\|u_{n}\right\|_{L^{t}} \rightarrow 0 .
$$

Proof. We set for $j \in \mathbb{Z}^{N}$

$$
Q_{j}=A\left(j+Q^{0}\right)=\left\{A j+A q: q \in Q^{0}\right\}
$$

where $Q^{0}$ is now the cube defined as follows

$$
Q^{0}=\left\{\left(x_{1}, . ., x_{n}\right) \in \mathbb{R}^{N}: 0 \leq x_{i}<1\right\} .
$$

Now let $x \in \mathbb{R}^{N}$ and set $y=A^{-1}(x)$. Clearly there exist $q \in Q^{0}$ and $j \in \mathbb{Z}^{N}$ such that $y=j+q$. So

$$
x=A y=A(j+q) \in Q_{j} .
$$

Then we conclude that

$$
\mathbb{R}^{N}=\bigcup_{j} Q_{j}
$$

Let $u_{n}$ be a bounded sequence in $H^{1}\left(\mathbb{R}^{N}\right)$ such that, up to a subsequence, $\left\|u_{n}\right\|_{L^{t}} \geq a>0$. We need to show that $u_{n}$ is non vanishing. Then, if $C$ is the 
constant for the Sobolev embedding $H^{1}\left(Q_{j}\right) \subset L^{t}\left(Q_{j}\right)$ and $\left\|u_{n}\right\|_{H^{1}}^{2} \leq M$, we have

$$
\begin{aligned}
0 & <a^{t} \leq \int\left|u_{n}\right|^{t}=\sum_{j} \int_{Q_{j}}\left|u_{n}\right|^{t}=\sum_{j}\left\|u_{n}\right\|_{L^{t}\left(Q_{j}\right)}^{t-2}\left\|u_{n}\right\|_{L^{t}\left(Q_{j}\right)}^{2} \\
& \leq\left(\sup _{j}\left\|u_{n}\right\|_{L^{t}\left(Q_{j}\right)}^{t-2}\right) \cdot \sum_{j}\left\|u_{n}\right\|_{L^{t}\left(Q_{j}\right)}^{2} \\
& \leq C\left(\sup _{j}\left\|u_{n}\right\|_{L^{t}\left(Q_{j}\right)}^{t-2}\right) \cdot \sum_{j}\left\|u_{n}\right\|_{H^{1}\left(Q_{j}\right)}^{2} \\
& =C\left(\sup _{j}\left\|u_{n}\right\|_{L^{t}\left(Q_{j}\right)}^{t-2}\right)\left\|u_{n}\right\|_{H^{1}}^{2} \leq C M\left(\sup _{j}\left\|u_{n}\right\|_{L^{t}\left(Q_{j}\right)}^{t-2}\right) .
\end{aligned}
$$

Then

$$
\left(\sup _{j}\left\|u_{n}\right\|_{L^{t}\left(Q_{j}\right)}\right) \geq\left(\frac{a^{t}}{C M}\right)^{1 /(t-2)}
$$

Then, for any $n$, there exists $j_{n} \in \mathbb{Z}^{N}$ such that

$$
\left\|u_{n}\right\|_{L^{t}\left(Q_{j_{n}}\right)} \geq \alpha>0 \text {. }
$$

Then, if we set $Q=A Q^{0}$, we easily have

$$
\left\|T_{j_{n}} u_{n}\right\|_{L^{t}(Q)}=\left\|u_{n}\right\|_{L^{t}\left(Q_{j_{n}}\right)} \geq \alpha>0 .
$$

Since $u_{n}$ is bounded, also $T_{j_{n}} u_{n}$ is bounded in $H^{1}\left(\mathbb{R}^{N}\right)$. Then we have, up to a subsequence, that $T_{j_{n}} u_{n} \rightarrow u_{0}$ weakly in $H^{1}\left(\mathbb{R}^{N}\right)$ and hence strongly in $L^{t}(Q)$. By (96), $u_{0} \neq 0$.

Lemma 33 Assumption (3) is satisfied namely

$$
\inf _{u \in H^{1}\left(R^{N}\right)} \Lambda(u)<\Lambda_{0}
$$

Proof. This lemma is analogous to lemma 19, however in this case the proof is easier: since $X=H^{1}\left(\mathbb{R}^{N}\right)$, we need only to construct a function $u \in H^{1}\left(\mathbb{R}^{N}\right)$ such that $\Lambda(u)<\Lambda_{0}$.

Such a function can be constructed as follows. Set

$$
u_{R}=\left\{\begin{array}{cc}
s_{0} & \text { if }|x|<R \\
0 & \text { if }|x|>R+1 \\
\frac{|x|}{R} s_{0}-(|x|-R) \frac{R+1}{R} s_{0} & \text { if } R<|x|<R+1
\end{array} .\right.
$$

Then

$$
\int\left|\nabla u_{R}\right|^{2} d x=O\left(R^{N-1}\right), \int\left|u_{R}\right|^{2} d x=O\left(R^{N}\right)
$$

so that 


$$
\frac{\int\left[\left|\nabla u_{R}\right|^{2}+2 V u_{R}^{2}\right] d x}{2 \int u_{R}^{2}} \leq \sup V+O\left(\frac{1}{R}\right) .
$$

Moreover

$$
\int W\left(u_{R}\right) d x=W\left(s_{0}\right) \operatorname{meas}\left(B_{R}\right)+\int_{B_{R+1} \backslash B_{R}} W\left(u_{R}\right) .
$$

So

$$
\begin{gathered}
\frac{\int W\left(u_{R}\right) d x}{\int u_{R}^{2}} \leq \frac{W\left(s_{0}\right) \operatorname{meas}\left(B_{R}\right)+c_{1} R^{N-1}}{\int u_{\lambda}^{2}} \leq\left(\text { since } W\left(s_{0}\right)<0\right) \\
\leq \frac{W\left(s_{0}\right) \text { meas }\left(B_{R}\right)}{s_{0}^{2} \text { meas }\left(B_{R+1}\right)}+\frac{c_{2} R^{N-1}}{R^{N}}=\frac{W\left(s_{0}\right)}{s_{0}^{2}}\left(\frac{R}{R+1}\right)^{N}+\frac{c_{2}}{R} .
\end{gathered}
$$

Then, by (97) e (98) we get

$$
\Lambda\left(u_{R}\right) \leq \sup V+\frac{W\left(s_{0}\right)}{s_{0}^{2}}\left(\frac{R}{R+1}\right)^{N}+O\left(\frac{1}{R}\right) .
$$

By lemma 17 we have $\inf V \leq \liminf _{\|u\|_{L^{t}} \rightarrow 0} \Lambda(u)$, then

$$
\Lambda\left(u_{R}\right) \leq \liminf _{\|u\|_{L^{t}} \rightarrow 0} \Lambda(u)+\sup V-\inf V+\frac{W\left(s_{0}\right)}{s_{0}^{2}}\left(\frac{R}{R+1}\right)^{N}+O\left(\frac{1}{R}\right)
$$

On the other hand, since by Lemma 18 the $L^{t}$ norm satisfies the property (5), we have by (6) that

$$
\liminf _{\|u\|_{L^{t}} \rightarrow 0} \Lambda(u) \leq \Lambda_{0}
$$

Clearly (99), (100) and assumption $\left(\mathrm{W}_{3}\right)$ imply that for $R$ large we have

$$
\Lambda\left(u_{R}\right)<\Lambda_{0}
$$

Then assumption (3) is satisfied.

Proof of Th. 29. The proof follows from Th. 26 and Lemmas 30, 31 and 33.

\section{Existence of Solitons for the nonlinear Klein- Gordon equation}

In this section we shall apply the abstract theorem 26 to the existence of hylomorphic solitons in $\mathbb{R}^{N}$ for the nonlinear Klein-Gordon equation (NKG). There are well known results on the existence of stable solutions for (NKG) ([27, [23]) 
and more recently the existence of hylomorphic solitons for (NKG) has been studied in [4] and 2 .

More exactly, we consider the equation

$$
\square \psi+W^{\prime}(\psi)=0
$$

where $\square=\partial_{t}^{2}-\Delta, \psi: \mathbb{R} \times \mathbb{R}^{N} \rightarrow \mathbb{C}(N \geq 3), W: \mathbb{C} \rightarrow \mathbb{R}$ and $W^{\prime}$ are as in (27) (see the beginning of section 3.1). Assume that

$$
W(s)=\frac{1}{2} m^{2} s^{2}+N(s), \quad s \geq 0, m \neq 0
$$

where $N(s)=o\left(s^{2}\right)$.

We make the following assumptions on $W$ :

- (NKG-i) (Positivity) $W(s) \geq 0$ for $s \geq 0$

- (NKG-ii) (Hylomorphy) $\exists s_{0} \in \mathbb{R}^{+}$such that $W\left(s_{0}\right)<\frac{1}{2} m^{2} s_{0}^{2}$

- (NKG-iii)(Growth condition) there are constants $c_{1}, c_{2}>0,2<r, q<$ $2 N /(N-2)$ such that for any $s>0$ :

$$
\left|N^{\prime}(s)\right| \leq c_{1} s^{r-1}+c_{2} s^{q-1} .
$$

We shall assume that the initial value problem is well posed for (NKG). Eq. (NKG) is the Euler-Lagrange equation of the action functional

$$
\mathcal{S}(\psi)=\int\left(\frac{1}{2}\left|\partial_{t} \psi\right|^{2}-\frac{1}{2}|\nabla \psi|^{2}-W(\psi)\right) d x d t .
$$

The energy and the charge take the following form:

$$
\begin{gathered}
E(\psi)=\int\left[\frac{1}{2}\left|\partial_{t} \psi\right|^{2}+\frac{1}{2}|\nabla \psi|^{2}+W(\psi)\right] d x \\
C(\psi)=-\operatorname{Re} \int i \partial_{t} \psi \bar{\psi} d x
\end{gathered}
$$

(the sign "minus" in front of the integral is a useful convention).

\subsection{The NKG as a dynamical system}

We set

$$
X=H^{1}\left(\mathbb{R}^{N}, \mathbb{C}\right) \times L^{2}\left(\mathbb{R}^{N}, \mathbb{C}\right)
$$

and we will denote the generic element of $X$ by $\mathbf{u}=(\psi(x), \hat{\psi}(x))$; then, by the well posedness assumption, for every $\mathbf{u} \in X$, there is a unique solution $\psi(t, x)$ of (NKG) such that

$$
\begin{aligned}
\psi(0, x) & =\psi(x) \\
\partial_{t} \psi(0, x) & =\hat{\psi}(x) .
\end{aligned}
$$


Using this notation, we can write equation (NKG) in Hamiltonian form:

$$
\begin{aligned}
& \partial_{t} \psi=\hat{\psi} \\
& \partial_{t} \hat{\psi}=\Delta \psi-W^{\prime}(\psi) .
\end{aligned}
$$

The time evolution map $\gamma: \mathbb{R} \times X \rightarrow X$ is defined by

$$
\gamma_{t} \mathbf{u}_{0}(x)=\mathbf{u}(t, x)
$$

where $\mathbf{u}_{0}(x)=(\psi(x), \hat{\psi}(x)) \in X$ and $\mathbf{u}(t, x)=(\psi(t, x), \hat{\psi}(t, x))$ is the unique solution of (106) and (107) satisfying the initial conditions (105). The energy and the charge, as functionals defined in $X$, become

$$
\begin{gathered}
E(\mathbf{u})=\int\left[\frac{1}{2}|\hat{\psi}|^{2}+\frac{1}{2}|\nabla \psi|^{2}+W(\psi)\right] d x \\
C(\mathbf{u})=-\operatorname{Re} \int i \hat{\psi} \bar{\psi} d x .
\end{gathered}
$$

\subsection{Existence results for NKG}

The following Theorem holds:

Theorem 34 Assume that $W$ satisfies $(N K G-i), \ldots,(N K G$-iii). Then there exists $\bar{\delta}>0$ such that the dynamical system described by the equation (NKG) has a family $\mathbf{u}_{\delta}(\delta \in(0, \bar{\delta}))$ of hylomorphic solitons.

The proof of this theorem is based on the abstract theorem 26. In this case the energy $E$ and the hylenic charge $C$ have the form (103) and (104) respectively.

Assumption (EC-1) is clearly satisfied. $E$ and $C$ are invariant under translations, so assumption (EC-2) is satisfied with respect to the action $T_{z}$ of the group $G=\mathbb{R}^{N}$ where

$$
T_{z} \mathbf{u}(x)=\mathbf{u}(x+z), \quad z \in \mathbb{R}^{N} .
$$

It can be seen that the coercitivity assumption (EC-3) is satisfied with $a=0$. Arguing as in lemma 16 (replacing $W$ by $N$ ) it can be shown that also (EC-4) is satisfied, namely that $E$ and $C$ satisfy the splitting property.

It remains to prove (3). First of all we set:

$$
\|\mathbf{u}\|_{\sharp}=\|(\psi, \hat{\psi})\|_{\sharp}=\max \left(\|\psi\|_{L^{r}},\|\psi\|_{L^{q}}\right)
$$

where $r, q$ are introduced in (NKG-iii). With some abuse of notation we shall write $\max \left(\|\psi\|_{L^{r}},\|\psi\|_{L^{q}}\right)=\|\psi\|_{\sharp}$. 
Lemma 35 The norm $\|\mathbf{u}\|_{\sharp}$ satisfies the property (5), namely

$$
\left\{\mathbf{u}_{n} \text { is a vanishing sequence }\right\} \Rightarrow\left\|\psi_{n}\right\|_{\sharp} \rightarrow 0 .
$$

Proof. Let $\psi_{n}$ be a bounded sequence in $H^{1}\left(\mathbb{R}^{N}\right)$ such that, up to a subsequence, $\left\|\psi_{n}\right\|_{\sharp} \geq a>0$. We need to show that $\psi_{n}$ is non vanishing. May be taking a subsequence, we have that at least one of the following holds:

- (i) $\left\|\psi_{n}\right\|_{\sharp}=\left\|\psi_{n}\right\|_{L^{r}}$

- (ii) $\left\|\psi_{n}\right\|_{\sharp}=\left\|\psi_{n}\right\|_{L^{q}}$

Suppose that (i) holds. Then, we argue as il lemma 32 If (ii) holds, we argue in the same way replacing $r$ with $q$.

Now we set

$$
\Lambda_{\sharp}=\liminf _{\|\mathbf{u}\|_{\sharp} \rightarrow 0} \Lambda(\mathbf{u})=\lim _{\varepsilon \rightarrow 0} \inf \left\{\Lambda(\psi, \hat{\psi}) \mid \hat{\psi} \in L^{2} ; \psi \in H^{1} ;\|\psi\|_{\sharp}<\varepsilon\right\} .
$$

By remark 9 , we have that $\Lambda_{0} \geq \Lambda_{\sharp}$; so let us evaluate $\Lambda_{\sharp}$.

Lemma 36 If $W$ satisfies assumption (NKG-iii), then the following inequality holds

$$
\Lambda_{\sharp} \geq m .
$$

Proof. By (NKG-iii) we have

$$
\begin{aligned}
\left|\int N(|\psi|) d x\right| & \leq k_{1} \int|\psi|^{r}+k_{2} \int|\psi|^{q} \\
& \leq k_{1}\|\psi\|_{\sharp}^{r}+k_{2}\|\psi\|_{\sharp}^{q} .
\end{aligned}
$$

If we assume that $\|\psi\|_{\sharp}=1$,

$$
\left|\int N(|\varepsilon \psi|) d x\right| \leq k_{1} \varepsilon^{r}+k_{2} \varepsilon^{q} .
$$

Now, choose $s \in(2, \min (r, q))$. Thus, if $\varepsilon>0$ is small enough, since $r, q>s>2$, we have

$$
\begin{aligned}
& \varepsilon^{s} \int\left(|\nabla \psi|^{2}+m^{2}|\psi|^{2}\right) d x-\left|\int N(|\varepsilon \psi|) d x\right| \\
& \geq \varepsilon^{s} k_{3}\|\psi\|_{\sharp}^{2}-k_{1} \varepsilon^{r}-k_{2} \varepsilon^{q}=k_{3} \varepsilon^{s}-k_{1} \varepsilon^{r}-k_{2} \varepsilon^{q} \geq 0
\end{aligned}
$$

and so

$$
\left|\int N(\varepsilon|\psi|) d x\right| \leq \varepsilon^{s} \int\left(|\nabla \psi|^{2}+m^{2}|\psi|^{2}\right) d x .
$$

Now we clearly have 


$$
\Lambda_{\sharp}=\lim _{\varepsilon \rightarrow 0} \inf \left\{\Lambda(\varepsilon \psi, \hat{\psi}) \mid \hat{\psi} \in L^{2}, \psi \in H^{1},\|\psi\|_{\sharp}=1\right\} .
$$

Let us estimate $\Lambda(\varepsilon \psi, \hat{\psi})$ using (110):

$$
\begin{aligned}
\Lambda(\varepsilon \psi, \hat{\psi}) & =\frac{\frac{1}{2} \int\left(|\hat{\psi}|^{2}+|\nabla \varepsilon \psi|^{2}+m^{2}|\varepsilon \psi|^{2}\right) d x+\int N(|\varepsilon \psi|) d x}{\left|\operatorname{Re} \int i \hat{\psi} \varepsilon \bar{\psi} d x\right|} \\
& \geq \frac{\frac{1}{2} \int|\hat{\psi}|^{2}+\left(\frac{\varepsilon^{2}}{2}-\varepsilon^{s}\right) \int\left(|\nabla \psi|^{2}+m^{2}|\psi|^{2}\right)}{\varepsilon\left|\operatorname{Re} \int i \hat{\psi} \bar{\psi} d x\right|} \\
& \geq \frac{\frac{1}{2} \int|\hat{\psi}|^{2}+\frac{\varepsilon^{2}}{2}\left(1-2 \varepsilon^{s-2}\right) m^{2} \int|\psi|^{2}}{\varepsilon\left(\int|\hat{\psi}|^{2} d x\right)^{1 / 2}\left(\int|\psi|^{2} d x\right)^{1 / 2}} \\
& \geq \frac{\left(\int|\hat{\psi}|^{2} d x\right)^{1 / 2} \cdot \varepsilon m \sqrt{1-2 \varepsilon^{s-2}}\left(\int|\psi|^{2} d x\right)^{1 / 2}}{\varepsilon\left(\int|\hat{\psi}|^{2} d x\right)^{1 / 2}\left(\int|\psi|^{2} d x\right)^{1 / 2}}=m \sqrt{1-2 \varepsilon^{s-2}}
\end{aligned}
$$

Then

$$
\lim \Lambda(\varepsilon \psi, \hat{\psi}) \geq m .
$$

So the conclusion follows by (111) and (112).

Next we will show that the hylomorphy assumption (3) is satisfied.

Lemma 37 Assume that $W$ satisfies $(N K G-i), \ldots,(N K G$-iii), then

$$
\inf _{\mathbf{u} \in X} \Lambda(\mathbf{u})<\Lambda_{0} .
$$

Proof. Let $R>0$; set

$$
u_{R}=\left\{\begin{array}{cc}
s_{0} & \text { if }|x|<R \\
0 & \text { if }|x|>R+1 \\
\frac{|x|}{R} s_{0}-(|x|-R) \frac{R+1}{R} s_{0} & \text { if } R<|x|<R+1
\end{array} .\right.
$$

By (NKG-ii) there exists $0<\beta<m$ such that

$$
W\left(s_{0}\right) \leq \frac{\beta^{2} s_{0}^{2}}{2} .
$$

We set $\psi=u_{R}$, and $\hat{\psi}=\beta u_{R}$. 
Then

$$
\begin{aligned}
\inf _{\mathbf{u} \in X} \Lambda(\mathbf{u}) & =\inf _{\psi, \hat{\psi}} \frac{\int\left(\frac{1}{2}|\hat{\psi}|^{2}+\frac{1}{2}|\nabla \psi|^{2}+W(\psi)\right) d x}{\left|\operatorname{Re} \int i \hat{\psi} \bar{\psi} d x\right|} \\
& \leq \frac{\int\left(\frac{1}{2} \beta^{2}\left|u_{R}\right|^{2}+\frac{1}{2}\left|\nabla u_{R}\right|^{2}+W\left(u_{R}\right)\right) d x}{\beta \int\left|u_{R}\right|^{2} d x} \\
& \leq \frac{\int_{|x|<R}\left(\frac{1}{2} \beta^{2}\left|u_{R}\right|^{2}+W\left(u_{R}\right)\right) d x}{\beta \int_{|x|<R}\left|u_{R}\right|^{2} d x} \\
& +\frac{\int_{R<|x|<R+1}\left(\frac{1}{2} \beta^{2}\left|u_{R}\right|^{2}+\frac{1}{2}\left|\nabla u_{R}\right|^{2}+W\left(u_{R}\right)\right) d x}{\beta \int_{|x|<R}\left|u_{R}\right|^{2} d x} \\
& =\frac{1}{2} \beta+\frac{\int_{|x|<R} W\left(s_{0}\right) d x}{\beta \int_{|x|<R}\left|s_{0}\right|^{2} d x}+O\left(\frac{1}{R}\right) .
\end{aligned}
$$

Then, by (114), we have

$$
\inf _{\mathbf{u} \in X} \Lambda(\mathbf{u}) \leq \frac{1}{2} \beta+\frac{\int_{|x|<R} \frac{1}{2} s_{0}^{2} \beta^{2}}{\beta \int_{|x|<R}\left|s_{0}\right|^{2} d x}+O\left(\frac{1}{R}\right)=\beta+O\left(\frac{1}{R}\right) .
$$

So

$$
\inf _{\mathbf{u} \in X} \Lambda(\mathbf{u}) \leq \beta .
$$

Then, since $\beta<m$, by remark 9, lemma 36 and (115), we have that

$$
\Lambda_{0} \geq \Lambda_{\sharp} \geq m>\beta \geq \inf _{\mathbf{u} \in X} \Lambda(\mathbf{u})
$$

and so the conclusion easily follows.

Proof of Th. 34, The assumptions (EC-1),...,(EC-4) and (3) are satisfied, then the proof follows by using Th. 26.

We conclude this section with the following theorem which gives some more information on the structure of the solitons:

Theorem 38 Let $\mathbf{u}$ be a hylomorphic soliton relative to the equation (NKG) with initial data $\mathbf{u}_{0}(x)=\left(\psi_{0}(x), \hat{\psi}_{0}(x)\right) \in X$. Then there exists $\omega \in \mathbb{R}$ such that $\psi_{0}$ satisfies the equation

$$
\begin{gathered}
-\Delta \psi_{0}+W^{\prime}\left(\psi_{0}\right)=\omega^{2} \psi_{0}, \\
\hat{\psi}_{0}=-i \omega \psi_{0}
\end{gathered}
$$

and

$$
\gamma_{t} \mathbf{u}_{0}(x)=\left[\begin{array}{c}
\psi_{0}(x) e^{-i \omega t} \\
-i \omega \psi_{0}(x) e^{-i \omega t}
\end{array}\right]
$$


Proof. Since $\mathbf{u}$ is a hylomorphic soliton it is a critical point of $E$ constrained on the manifold $\mathfrak{M}_{c}=\{\mathbf{u} \in X: C(\mathbf{u})=c\}$.

Clearly

$$
E^{\prime}\left(u_{0}\right)=-\omega C^{\prime}\left(u_{0}\right)
$$

where $-\omega$ is a Lagrange multiplier. We now compute the derivatives $E^{\prime}\left(u_{0}\right), C^{\prime}\left(u_{0}\right)$. For all $\left(v_{0}, v_{1}\right) \in X=H^{1}\left(\mathbb{R}^{N}, \mathbb{C}\right) \times L^{2}\left(\mathbb{R}^{N}, \mathbb{C}\right)$, we have

$$
\begin{aligned}
& E^{\prime}\left(u_{0}\right)\left[\begin{array}{l}
v_{0} \\
v_{1}
\end{array}\right]=\operatorname{Re} \int\left[\hat{\psi}_{0} \overline{v_{1}}+\nabla \psi_{0} \overline{\nabla v_{0}}+W^{\prime}\left(x, \psi_{0}\right) \overline{v_{0}}\right] d x \\
& C^{\prime}\left(u_{0}\right)\left[\begin{array}{l}
v_{0} \\
v_{1}
\end{array}\right]=-\operatorname{Re} \int\left(i \hat{\psi}_{0} \overline{v_{0}}+i v_{1} \overline{\psi_{0}}\right) d x \\
&=-\operatorname{Re} \int\left(i \hat{\psi}_{0} \overline{v_{0}}+\overline{i v_{1} \overline{\psi_{0}}}\right) d x \\
&=-\operatorname{Re} \int\left(i \hat{\psi}_{0} \overline{v_{0}}-i \psi_{0} \overline{v_{1}}\right) d x .
\end{aligned}
$$

Then (118) can be written as follows:

$$
\begin{aligned}
\operatorname{Re} \int\left[\nabla \psi_{0} \overline{\nabla v_{0}}+W^{\prime}\left(x, \psi_{0}\right) \overline{v_{0}}\right] d x & =\omega \operatorname{Re} \int i \hat{\psi}_{0} \overline{v_{0}} d x \\
\operatorname{Re} \int \hat{\psi}_{0} \overline{v_{1}} d x & =-\omega \operatorname{Re} \int i \psi_{0} \overline{v_{1}} d x .
\end{aligned}
$$

Then

$$
\begin{aligned}
-\Delta \psi_{0}+W^{\prime}\left(x, \psi_{0}\right) & =i \omega \hat{\psi}_{0} \\
\hat{\psi}_{0} & =-i \omega \psi_{0} .
\end{aligned}
$$

So we get (116). From (116) and (119), we easily verify that (117) solves (106), (107).

\section{References}

[1] Abrikosov A.A., On the magnetic properties of superconductors of the second group, Sov. Phys. JETP 5 (1957), 1174-1182.

[2] Badiale M., Benci V., Rolando S., A nonlinear elliptic equation with singular potential and applications to nonlinear field equations, J. Eur. Math. Soc. 9 (2007), 355-381.

[3] Badiale M., Benci V., Rolando S., Three dimensional vortices in the nonlinear wave equation, Bollettino U.M.I., Serie 9, II (2009), 105-134. 
[4] Bellazzini J., Benci V., Bonanno C., Micheletti A.M., Solitons for the Nonlinear Klein-Gordon-Equation, Advances in Nonlinear Studies, 10, (2010), 481-500 (arXiv:0712.1103).

[5] Bellazzini J., Benci V., Bonanno C., Sinibaldi E., Hylomorphic solitons in the nonlinear Klein-Gordon equation, Dynamics of Partial Differential Equations, 6 (2009), 311-333. (arXiv:0810.5079).

[6] Bellazzini J., Benci V., Ghimenti M., Micheletti A.M., On the existence of the fundamental eigenvalue of an elliptic problem in $\mathbb{R}^{N}$, Adv. Nonlinear Stud. 7 (2007), 439-458.

[7] Bellazzini J., Bonanno C., Nonlinear Schrödinger equations with strongly singular potentials. Proceedings of the Royal Society of Edinburgh: Section A Mathematics 140: (2010), 707-721.

[8] Bellazzini J., Bonanno C., Siciliano G., Magnetostatic vortices in two dimensional Abelian gauge theory, Mediterranean J. Math., Mediterranean Journal of Mathematics, 6 (2009), 347-366 .

[9] Benci V, Hylomorphic solitons, Milan J. Math. 77 (2009), 271-332.

[10] Benci V. Fortunato D., Three dimensional vortices in Abelian Gauge Theories, Nonlinear Analysis T.M.A., 70 (2009), 4402-4421.

[11] Benci V., Fortunato D., Hylomorphic Solitons on lattices, Discrete and continuous dynamical systems, 28 (2010) pp. 875-897.

[12] Benci V., Fortunato D., On the existence of stable charged Q-balls, Journal of Mathematical Physics, to appear. (ArXive. 1011.5044).

[13] Benci V. Fortunato D., Hamiltonian formulation of the Klein-GordomMaxwell equations, Rend. Lincei Mat. Appl. 22 (2011), 1-22..

[14] Benci V., Fortunato D., Existence of solitons in nonlinear beam equation, (ArXive 1102.5315).

[15] Benci V. Fortunato D., Existence of hylomorphic solitary waves in Klein-Gordon and in Klein-Gordon-Maxwell equations, Rend. Lincei Mat. Appl.20 (2009), 243-279. (arXiv:0903.3508).

[16] Benci V. Fortunato D., Spinning Q-balls for the Klein-Gordon-Maxwell equations, Comm. Math. Phys., 295 (2010), 639-668.

[17] Benci V, Ghimenti M., Micheletti A.M., The Nonlinear Schroedinger equation: solitons dynamics, Journal of Differential Equations 249, (2010), 3312-3341 (arXiv:0812.4152).

[18] Benci V., Visciglia N., Solitary waves with non vanishing angular momentum, Adv. Nonlinear Stud. 3 (2003), 151-160. 
[19] Buslaev V. S., Sulem C., On asymptotic stability of solitary waves for nonlinear Schrödinger equations. Annales de l'institut Henri Poincaré (C) Analyse non linéaire, 20 (2003), 419-475.

[20] Cazenave T., Lions P.L. , Orbital stability of standing waves for some nonlinear Schrödinger equations, Comm. Math. Phys. 85 (1982), no. 4, $549-561$.

[21] Esteban M., Lions P.L. A compactness lemma, Nonlinear Analysis, 7 (1983), 381-385.

[22] Gelfand I.M., Fomin S.V., Calculus of Variations, Englewood Cliffs, NJ. Prentice-Hall, (1963).

[23] Grillakis M., Shatah J,, Strauss W., Stability theory of solitary waves in the presence of symmetry, I, J. Funct. Anal. 74 (1987), 160-197.

[24] Kim C.,Kim S.,Kim Y.Global nontopological vortices, Phys. Review D, 47, (1985), 5434-5443.

[25] Nielsen H., Olesen P., Vortex-line models for dual strings, Nucl. Phys. B 61, (1973), 45-61.

[26] Palais R.S., The principle of symmetric criticality, Comm. Math. Phys., 69, (1979), 19-30.

[27] Shatah J. , Stable Standing waves of Nonlinear Klein-Gordon Equations, Comm. Math. Phys., 91, (1983), 313-327.

[28] Vilenkin A., Shellard E.P.S., Cosmic strings and other topological defects, Cambrige University press, Cambridge (1994).

[29] Volkov M.S., Existence of spinning solitons in field theory, arXiv:hep-th/0401030 (2004)

[30] Volkov M.S., Wöhnert E., Spinning Q-balls, Phys. Rev. D 66 (2002) 085003. 